\title{
THE DIRICHLET PROBLEM FOR A COMPLEX HESSIAN EQUATION ON COMPACT HERMITIAN MANIFOLDS WITH BOUNDARY
}

\author{
DONGWEI GU AND NGOC CUONG NGUYEN
}

\begin{abstract}
We solve the classical Dirichlet problem for a general complex Hessian equation on a small ball in $\mathbb{C}^{n}$. Then, we show that there is a continuous solution, in pluripotential theory sense, to the Dirichlet problem on compact Hermitian manifolds with boundary that equipped locally conformal Kähler metrics, provided a subsolution.
\end{abstract}

\section{INTRODUCTION}

Let $(\bar{M}, \alpha)$ be a compact Hermitian manifold with smooth boundary $\partial M$, of complex dimension $n$. Let us denote $M:=\bar{M} \backslash \partial M$. Let $1 \leq m \leq n$ be an integer. Fix a real $(1,1)$-form $\chi$ on $\bar{M}$. We have given a right hand side $f \in C^{\infty}(\bar{M})$ positive and a smooth boundary data $\varphi \in C^{\infty}(\partial M)$. The classical Dirichlet problem for the complex Hessian equation is to find a real-valued function $u \in C^{\infty}(\bar{M})$ :

$$
\begin{aligned}
& \left(\chi+d d^{c} u\right)^{m} \wedge \alpha^{n-m}=f \alpha^{n}, \\
& u=\varphi \quad \text { on } \partial M,
\end{aligned}
$$

where $u$ is subjected to point-wise inequalities

$$
\left(\chi+d d^{c} u\right)^{k} \wedge \alpha^{n-k}>0, \quad k=1, . ., m .
$$

We first solve the equation in a small ball.

Theorem 1.1. Let $M=B(z, \delta) \subset \subset B(0,1)$ be a Euclidean ball of radius $\delta$ in the unit ball $B(0,1) \subset \mathbb{C}^{n}$. Assume that $\chi, \alpha$ are smooth on $\overline{B(0,1)}$. Then, the classical Dirichlet problem (1.1) is uniquely solvable for $\delta$ small enough, which depends only on $\chi, \alpha$.

A $C^{2}$ real-valued function satisfying inequalities (1.2) is called $(\chi, m)-\alpha$ - subharmonic. These inequalities can be generalised to non-smooth functions to obtain the class of $(\chi, m)-\alpha$-subharmonic functions on $M$. Locally, the convolution of a function in this class with a smooth kernel, in general, will not belong to this class again. However, using the theorem above and an adapted potential theory, we prove the approximation property.

Corollary 1.2. Any $(\chi, m)-\alpha$-subharmonic function on $M$ is locally approximated by a decreasing sequence of smooth $(\chi, m)-\alpha$-subharmonic functions.

2010 Mathematics Subject Classification. 53C55, 35J96, 32U40.

Key words and phrases. Dirichlet problem, weak solutions, Hessian equation, Hermitian manifold. 
Following Bedford-Taylor [1, 2, 3] and Kołodziej [38, 39, 40, the two results above allow us to use Perron's envelope together with pluripotential theory techniques, adapted to this setting, to study weak solutions to this equation with the continuous right hand sides. A Hermitian metric $\alpha$ is called a locally conformal Kähler metric on $M$ if at every given point on $M$, there exist a local chart $\Omega$ and a smooth realvalued function $G$ such that $e^{G} \alpha$ is Kähler on $\Omega$. This class of metric is strictly larger than the Kähler one, and not every Hermitian metric is locally conformal Käher (see e.g. 9]). Our main result is

Theorem 1.3. Assume that $\alpha$ is locally conformal Kähler. Let $0 \leq f \in C^{0}(\bar{M})$ and $\varphi \in C^{0}(\partial M)$. Assume that there is a $C^{2}$-subsolution $\rho$, i.e., $\rho$ satisfying inequalities (1.2) and

$$
\left(\chi+d d^{c} \rho\right)^{m} \wedge \alpha^{n-m} \geq f \alpha^{n} \quad \text { in } \bar{M}, \quad \rho=\varphi \quad \text { on } \partial M .
$$

Then, there exists a continuous solution to the Dirichlet problem (1.1) in pluripotential theory sense.

When $m=n$ we need not assume $\alpha$ is locally conformal Kähler. The Dirichlet problem for the Monge-Ampère equation on compact Hermitian manifolds with boundary has been studied extensively, in smooth category, in recent years by Cherrier-Hanani [15, 16], Guan-Li [28] and Guan-Sun [29]. Our theorem generalises the result in 28 for continuous datum.

When $1<m<n$ and $\alpha=d d^{c}|z|^{2}$ is the Euclidean metric the Dirichlet problem for the complex Hessian equation in a domain in $\mathbb{C}^{n}$ has been studied extensively by many authors [5, 14, 18, 45, 48, 50, 54. To our best knowledge the classical Dirichlet problem (1.1) on a compact Hermitian (or Kähler) manifold with boundary still remains open. The difficulty lies in the $C^{1}$-estimate for a general Hermitian metric $\alpha$. Here we only obtain such an estimate in a small ball (Theorem 1.1). Moreover, in our approach, the locally conformal Kähler assumption of $\alpha$ is needed to define the complex Hessian operator of bounded functions (Section 3).

Motivations to study the Dirichlet problem for such equations come from recent developments of fully non-linear elliptic equations on compact complex manifolds. First, it is the natural problem after the complex Hessian equation was solved by Dinew-Kołodziej [19] on compact Kähler manifolds, and by Székelyhidi [62] and Zhang [69] on compact Hermitian manifolds. Indeed, such a question is raised in [62. Second, on compact Hermitian manifolds, it is strongly related to the elementary symmetric positive cone with which several types of equations associated were studied by Székelyhidi-Tosatti-Weinkove [63, Tosatti-Weinkove [65, 66]. Our results may provide some tools to study these cones. In the case when $\alpha$ is Kähler ( $\chi$ may be not), the Hessian type equations related to a Strominger system, which generalised Fu-Yau equations [24, have been studied recently by Phong-PicardZhang [56, 57, 58. Lastly, the viscosity solutions of fully nonlinear elliptic equations on Riemannian and Hermitian manifolds have been also investigated by Harvey and Lawson [32, 33] in a more general frame work, and the existence of continuous solutions was proved under additional assumptions on the relation of the group structure of manifolds and given equations.

Organisation. In Section 2 we give definitions for generalised $m$ - subharmonic functions and their basic properties. Assuming Theorem[1.1 in Section3 we develop "pluripotential theory" for corresponding generalised $m$ - subharmonic functions to the equation. This enable us to prove Corollary 1.2. Section 4 is devoted to study 
weak solutions to the Dirichlet problem in a small Euclidean ball. Theorem 1.3 is proved in Section 4.2. Finally, in Sections [5, 6, 7, 8 we prove Theorem 1.1 independent of the other sections. The appendix is given in Section 9 .

Acknowledgement. We are grateful to Sławomir Kołodziej for many valuable comments, which help to improve significantly the exposition of the paper. We also thank Sławomir Dinew who has read the draft version of our paper and pointed out some mistakes. Furthermore, we would like to thank Blaine Lawson for bringing our attention to results in [32, 33, 34. Part of this work was written during the visit of the second author at Tsinghua Sanya International Mathematics Forum in January 2016. He would like to thank the organisers for the invitation and the members of the institute for their hospitality. The second author is supported by NCN grant 2013/08/A/ST1/00312. The first author is supported by the Ideas Plus grant 0001/ID3/2014/63 of the Polish Ministry Of Science and Higher Education. Lastly, we would like to thank the referee for the helpful comments.

\section{Generalised $m$-Subharmonic Functions}

Fix a Hermitian metric $\alpha=\sqrt{-1} \alpha_{i \bar{j}} d z^{i} \wedge d \bar{z}^{j}$ on a bounded open set $\Omega$ in $\mathbb{C}^{n}$. Consider another real $(1,1)$-form $\chi=\sqrt{-1} \chi_{i \bar{j}} d z^{i} \wedge d \bar{z}^{j}$.

A $C^{2}$ function $u$ on $\Omega$ is called $\alpha$-subharmonic if

$$
\Delta_{\alpha} u(z)=\sum \alpha^{\bar{j} i}(z) \frac{\partial^{2} u}{\partial z^{i} \partial \bar{z}^{j}}(z) \geq 0,
$$

where $\alpha^{\bar{j} i}$ is the inverse of $\alpha_{i \bar{j}}$. We can rewrite it simply in term of $(n, n)$-positive forms

$$
d d^{c} u \wedge \alpha^{n-1} \geq 0, \quad \text { where } d d^{c}=\frac{\sqrt{-1}}{\pi} \partial \bar{\partial} .
$$

This form has the advantage that one can generalise to non-smooth functions and with possibility define higher power of the wedge product of $d d^{c} u$ (see Remark 2.5). We start with the following definition which is adapted from subharmonic functions.

Definition 2.1. A function $u: \Omega \rightarrow[-\infty,+\infty[$ is called $\alpha$-subharmonic if

(a) $u$ is upper semicontinuous and $u \in L_{l o c}^{1}(\Omega)$;

(b) for every relatively compact open set $D \subset \subset \Omega$ and every $h \in C^{0}(\bar{D})$ and $\Delta_{\alpha} h=0$ in $D$, if $h \geq u$ on $\partial D$, then $h \geq u$ on $\bar{D}$.

Remark 2.2. Comparing to subharmonic functions we have that

(1) If an upper semicontinuous $u$ satisfies (b), then by Harvey-Lawson [31, Theorem 9.3(A)] it follows that either $u \equiv-\infty$ or $u \in L_{l o c}^{1}(\Omega)$.

(2) The $\alpha$-subharmonicity for continuous function $u$ is equivalent to the inequality $\Delta_{\alpha} u \geq 0$ in the distributional sense, a detailed statement of this fact will be given in Lemma 9.10 (Appendix).

We shall define $(\chi, m)-\alpha$-subharmonicity for non-smooth functions. Let us denote

$$
\Gamma_{m}=\left\{\lambda=\left(\lambda_{1}, \ldots, \lambda_{n}\right) \in \mathbb{R}^{n}: S_{1}(\lambda)>0, \ldots, S_{m}(\lambda)>0\right\} .
$$

The positive cone $\Gamma_{m}(\alpha)$ associated with the metric $\alpha$ is defined as follows.

$$
\Gamma_{m}(\alpha)=\left\{\gamma \text { real }(1,1)-\text { form: } \gamma^{k} \wedge \alpha^{n-k}>0 \text { for every } k=1, \ldots, m\right\} .
$$


In other words, in the orthonormal coordinate such that $\alpha=\sum_{i} \sqrt{-1} d z^{i} \wedge d \bar{z}^{i}$ at a given point in $\Omega$, and $\gamma=\sum_{i} \lambda_{i} \sqrt{-1} d z^{i} \wedge d \bar{z}^{i}$ also diagonalised at this point, then $\gamma \in \Gamma_{m}(\alpha)$ if $\left(\lambda_{1}, \ldots, \lambda_{n}\right) \in \Gamma_{m}$.

Definition 2.3. A function $u: \Omega \rightarrow[-\infty,+\infty[$ is called $m-\alpha$-subharmonic if $u$ is $\tilde{\alpha}$-subharmonic for any $\tilde{\alpha}$ of the form $\tilde{\alpha}^{n-1}=\gamma_{1} \wedge \cdots \wedge \gamma_{m-1} \wedge \alpha^{n-m}$, where $\gamma_{1}, \ldots, \gamma_{m-1} \in \Gamma_{m}(\alpha)$.

Here, the metric $\tilde{\alpha}$ is uniquely defined thanks to a result of Michelsohn [52]. By a simple consideration we have a generalisation

Definition 2.4. A function $u: \Omega \rightarrow[-\infty,+\infty[$ is called $(\chi, m)-\alpha$ - subharmonic if $u+\rho$ is $\tilde{\alpha}$-subharmonic for any $\tilde{\alpha}$ of the form $\tilde{\alpha}^{n-1}=\gamma_{1} \wedge \cdots \wedge \gamma_{m-1} \wedge \alpha^{n-m}$, where $\gamma_{1}, \ldots, \gamma_{m-1} \in \Gamma_{m}(\alpha)$, and the smooth function $\rho$ is defined, up to a constant, by the equation $d d^{c} \rho \wedge \tilde{\alpha}^{n-1}=\chi \wedge \tilde{\alpha}^{n-1}$.

Notice that when $\chi \equiv 0$, Definition 2.4 coincides with Definition 2.3. Thanks to Lemma 9.10 in Appendix, we get that for a $(\chi, m)-\alpha$-subharmonic function $u$,

$$
\left(\chi+d d^{c} u\right) \wedge \gamma_{1} \wedge \cdots \wedge \gamma_{m-1} \wedge \alpha^{n-m} \geq 0
$$

for any collection $\gamma_{i} \in \Gamma_{m}(\alpha)$, in the weak sense of currents. We denote the set of all $(\chi, m)-\alpha$-subharmonic functions in $\Omega$ by

$$
S H_{\chi, m}(\alpha, \Omega) \quad \text { or } S H_{\chi, m}(\alpha)
$$

(for short) if the considered set is clear from the context.

Remark 2.5. (1) For a $C^{2}$ function $u$ the inequality (2.3) is equivalent to the inequalities

$$
\left(\chi+d d^{c} u\right)^{k} \wedge \alpha^{n-k} \geq 0 \text { for } k=1, \ldots, m \text {. }
$$

This fact can be seen as follows: for any real $(1,1)$-form $\tau \in \Gamma_{m}(\alpha)$ and $1 \leq k \leq m$,

$$
\frac{\tau^{k} \wedge \alpha^{n-k}}{\alpha^{n}}=\left(\inf _{\gamma}\left\{\frac{\tau \wedge \gamma^{k-1} \wedge \alpha^{n-k}}{\alpha^{n}}\right\}\right)^{k},
$$

where $\gamma$ is taken such that $\gamma \in \Gamma_{m}(\alpha)$ and $\gamma^{k} \wedge \alpha^{n-k} / \alpha^{n}=1$. In other words, $u \in S H_{\chi, m}(\alpha, \Omega)$ if and only if $\chi+d d^{c} u \in \overline{\Gamma_{m}(\alpha)}$ at any given point in $\Omega$.

(2) There are another definitions for $m-\alpha$-subharmonic functions. The first one is suggested by Błocki [5] and the second one is given by Lu [49, Definition 2.3] in a more general setting. All definitions are equivalent in the case of $m$-subharmonic functions, i.e. $\alpha=d d^{c}|z|^{2}$. Later on, by Lemma 9.17, we will find that our definition is equivalent to the one in [49].

We list here some basic properties of $(\chi, m)-\alpha$-subharmonic functions.

Proposition 2.6. Let $\Omega$ be a bounded open set in $\mathbb{C}^{n}$.

(a) If $u_{1} \geq u_{2} \geq \cdots$ is a decreasing sequence of $(\chi, m)-\alpha$-subharmonic functions, then $u:=\lim _{j \rightarrow \infty} u_{j}$ is either $(\chi, m)-\alpha$-subharmonic or $\equiv-\infty$.

(b) If $u, v$ belong to $S H_{\chi, m}(\alpha)$, then so does $\max \{u, v\}$.

(c) Let $\left\{u_{\alpha}\right\}_{\alpha \in I} \subset S H_{\chi, m}(\alpha)$ be a family locally uniformly bounded above. Put $u(z):=\sup _{\alpha} u_{\alpha}(z)$. Then, the upper semicontinuous regularisation $u^{*}$ is $(\chi, m)-\alpha$-subharmonic. 
Proof. It is enought to verify $\tilde{\alpha}$-subharmonicity for every $\tilde{\alpha}^{n-1}=\gamma_{1} \wedge \cdots \wedge \gamma_{m-1} \wedge \alpha$ with $\gamma_{i} \in \Gamma_{m}(\alpha)$. Once $\tilde{\alpha}$ is fixed the proof follows from Appendix (Proposition 9.3. Corollary 9.16).

\section{Potential estimates in a small ball}

In this section we develop potential theory for $(\chi, m)-\alpha$-subharmonic functions in a Euclidean ball, where $\alpha$ is conformal to a Kähler metric on this ball. To do this we fix a ball $B:=B(z, r) \subset \subset \Omega$ with the small radius, where $\Omega$ is a bounded open set in $\mathbb{C}^{n}$. We also fix a smooth function $G: \bar{B} \rightarrow \mathbb{R}$ such that $\omega:=e^{G} \alpha$ is Kähler metric, i.e.,

$$
d\left(e^{G} \alpha\right)=0 \quad \text { on } \bar{B} .
$$

Notice that by Definition 2.4 we have $S H_{\chi, m}(\alpha) \equiv S H_{\chi, m}(\omega)$ as $\Gamma_{m}(\alpha) \equiv \Gamma_{m}(\omega)$.

First, we will work with an apparently smaller class of functions.

Definition 3.1. Let $v$ be a $(\chi, m)-\alpha$-subharmonic function in a neighborhood of $\bar{B} . v$ is said to belong to $\mathcal{A}$ if there exists sequence of smooth $(\chi, m)-\alpha$-subharmonic functions $v_{j} \in C^{\infty}(\bar{B})$ decreasing point-wise to $v$ in $B$ as $j$ goes to $\infty$.

For simplicity we also assume in this section that for every $z \in \bar{\Omega}$,

$$
\chi(z) \in \Gamma_{m}(\alpha),
$$

(otherwise we replace $\chi$ by $\tilde{\chi}:=\chi+C d d^{c} \rho$ for a strictly plurisubharmonic function $\rho$ in $\bar{\Omega}$ and $C>0$ large.) Since $\bar{B}$ is compact, there exist $0<c_{0} \leq 1$, depending on $\chi, \alpha, \bar{B}$, such that

$$
\chi-c_{0} \alpha \in \Gamma_{m}(\alpha) .
$$

Throughout the paper we often write

$$
\chi_{u}:=\chi+d d^{c} u \text { for } u \in S H_{\chi, m}(\alpha) .
$$

3.1. Hessian operators. According to the results in 43 for any $v_{1}, \ldots, v_{m} \in \mathcal{A} \cap$ $C^{0}(\bar{B})$, the wedge product

$$
\chi_{v_{1}} \wedge \cdots \wedge \chi_{v_{m}} \wedge \alpha^{n-m}
$$

is a well defined positive Radon measure for a general Hermitian metric $\alpha$. However, to define the wedge product for $v_{i} \in \mathcal{A} \cap L^{\infty}(\bar{B})$ we will need the Kähler property of $\omega=e^{G} \alpha$ in (3.1).

Following ideas of Bedford-Taylor 2], by a simple modification, we can define the wedge product for $v_{i} \in \mathcal{A} \cap L^{\infty}(\bar{B})$ as follows. Fix a strictly plurisubharmonic function $\varphi$ in a neighborhood of $\bar{B}$ such that

$$
\tau:=d d^{c} \varphi-\chi>0 \text {. }
$$

Let us denote $w_{i}:=v_{i}+\varphi$. Then $w_{i}$ is $m-\omega$-subharmonic and bounded in $\bar{B}$, which is also in the class $\mathcal{A}$. Since $\omega$ is Kähler, we define inductively for $1 \leq k \leq m$,

$$
d d^{c} w_{k} \wedge \cdots \wedge d d^{c} w_{1} \wedge \omega^{n-m}:=d d^{c}\left(w_{k} d d^{c} w_{k-1} \wedge \cdots \wedge d d^{c} w_{1} \wedge \omega^{n-m}\right) .
$$

The resulted wedge product is a positive $(n-m+k, n-m+k)-$ current. Then, one puts

$$
d d^{c} w_{k} \wedge \cdots \wedge d d^{c} w_{1} \wedge \alpha^{n-m}:=e^{(m-n) G} d d^{c} w_{k} \wedge \cdots \wedge d d^{c} w_{1} \wedge \omega^{n-m} .
$$


We see that local properties that hold for a positive current on the right hand side will be preserved to the positive currents on the left hand side. Finally, using a formal expansion, we set

$$
\begin{aligned}
& \chi_{v_{1}} \wedge \cdots \wedge \chi_{v_{m}} \wedge \alpha^{n-m} \\
& :=\sum_{\left\{i_{1}, \ldots, i_{k}\right\} \subset\{1, \ldots, m\}}(-1)^{n-k} d d^{c} w_{i_{1}} \wedge \cdots \wedge d d^{c} w_{i_{k}} \wedge \alpha^{n-m} \wedge \tau^{n-k} .
\end{aligned}
$$

This is an honest equality in the case $v_{i}^{\prime} s$ are smooth functions. The right hand side still makes sense, when $v_{i}^{\prime} s$ are only bounded, by (3.3) and (3.4). Thus, we get the wedge product on the left hand side is a well-defined $(n, n)$-positive current.

We also observe that the equation (3.5) does not depend on the choice of a strictly plurisubharmonic function $\varphi$ satisfying $d d^{c} \varphi-\chi>0$. Moreover, let $T=$ $\chi_{v_{1}} \wedge \cdots \wedge \chi_{v_{k}} \wedge \alpha^{n-m}$ for $v_{i} \in \mathcal{A} \cap L^{\infty}(\bar{B})$ and $w \in \mathcal{A} \cap L^{\infty}(\bar{B})$. Then, we have

$$
\left(\chi+d d^{c} w\right) \wedge T=\chi \wedge T+d d^{c} w \wedge T .
$$

In other words, the definition of the wedge product obeys the linearity as in the smooth case.

Remark 3.2. If we do not assume $d \alpha=0$ ( or $d\left(e^{G} \alpha\right)=0$ for some function $G$ ), then in the inductive definition we cannot get rid of the extra terms, e.g.,

$$
d d^{c} v_{k} \wedge \cdots \wedge d d^{c} v_{1} \wedge d d^{c} \alpha^{n-m} .
$$

As $d d^{c} v_{i}$ is not $(1,1)$-positive current, we do not know how to define the wedge product for bounded functions $v_{i}$ in $\mathcal{A}$ once the power of the base $\alpha$ is less than $n-m$. It is worth to mention that if $v_{i}^{\prime} s$ are continuous and belong to $\mathcal{A}$, then we can use the uniform convergence of potentials to define wedge product as in [43].

As in [43] the Chern-Levine-Nirenberg (CLN) inequalities are proved quickly in the present setting.

Lemma 3.3. Let $u_{1}, \ldots, u_{m} \in \mathcal{A} \cap L^{\infty}(\bar{B})$. Let $K \subset \subset B$ be a compact set. Then,

$$
\int_{K} \chi_{u_{1}} \wedge \cdots \wedge \chi_{u_{m}} \wedge \alpha^{n-m} \leq C
$$

where $C$ depends on $\alpha, K, B\left\|u_{1}\right\|_{L^{\infty}(B)}, \ldots,\left\|u_{m}\right\|_{L^{\infty}(B)}$.

Proof. Since $\omega=e^{G} \alpha$ is Kähler and $G$ is bounded on $\bar{B}$,

$$
\int_{K} \chi_{u_{1}} \wedge \cdots \wedge \chi_{u_{m}} \wedge \alpha^{n-m} \leq e^{(n-m) \sup _{\bar{B}}|G|} \int_{K} \chi_{u_{1}} \wedge \cdots \wedge \chi_{u_{m}} \wedge \omega^{n-m} .
$$

Hence, the inequality follows from formulas (3.4), (3.5) and the classical argument by integration by parts (see [43, Proposition 2.9]).

The following Bedford-Taylor convergence theorems are crucial in our approach.

Theorem 3.4. Let $\left\{u_{1}^{j}\right\}_{j \geq 1}, \ldots,\left\{u_{m}^{j}\right\}_{j \geq 1} \subset \mathcal{A} \cap L^{\infty}(\bar{B})$ be decreasing (or increasing) sequences which converge point-wise to $u_{1}, \ldots, u_{m} \in \mathcal{A} \cap L^{\infty}(\bar{B})$, respectively. Then, the sequence of positive measures

$$
\left(\chi+d d^{c} u_{1}^{j}\right) \wedge \cdots \wedge\left(\chi+d d^{c} u_{m}^{j}\right) \wedge \alpha^{n-m}
$$

converges weakly to the positive measure

$$
\left(\chi+d d^{c} u_{1}\right) \wedge \cdots \wedge\left(\chi+d d^{c} u_{m}\right) \wedge \alpha^{n-m}
$$

as $j \rightarrow \infty$. 
Proof. Recall that $\omega:=e^{G} \alpha$ is a Kähler form on $\bar{B}$. By definitions (3.4) and (3.5) it is enough to show that if decreasing sequences of bounded $m-\omega$-suharmonic functions $\left\{v_{1}^{j}\right\}_{j \geq 1}, \ldots,\left\{v_{m}^{j}\right\}_{j \geq 1}$ converge to bounded $m-\omega$-subharmonic functions $v_{1}, \ldots, v_{m}$, respectively, then the sequence of $(n, n)$ - positive currents $d d^{c} v_{1}^{j} \wedge \cdots \wedge$ $d d^{c} v_{m}^{j} \wedge \omega^{n-m}$ weakly converges to $d d^{c} v_{1} \wedge \cdots \wedge d d^{c} v_{m} \wedge \omega^{n-m}$. Therefore, the theorem follows by an easy adaption of arguments of Bedford-Taylor [2].

Let us define the notion capacity associated with Hessian operators which plays important role in the study of bounded $(\chi, m)-\alpha$-subharmonic functions. For a Borel set $E \subset B$,

$$
\operatorname{cap}(E):=\sup \left\{\int_{E}\left(\chi+d d^{c} v\right)^{m} \wedge \alpha^{n-m}: v \in \mathcal{A}, 0 \leq v \leq 1\right\} .
$$

We first observe that this capacity is equivalent to another capacity.

Lemma 3.5. For a Borel set $E \subset B$,

$$
\mathbf{c}_{m}(E):=\sup \left\{\int_{E}\left(d d^{c} w\right)^{m} \wedge \alpha^{n-m}: w \in \mathcal{A}_{0}, 0 \leq w \leq 1\right\},
$$

where $\mathcal{A}_{0}$ is the class $\mathcal{A}$ with $\chi \equiv 0$. Then, there exists a constant $C$ depending on $\chi, \alpha$ such that

for any Borel set $E \subset B$.

$$
\frac{1}{C} \operatorname{cap}(E) \leq \mathbf{c}_{m}(E) \leq C \operatorname{cap}(E)
$$

Proof. Since $\chi \leq d d^{c} \varphi$ for some smooth plurisubharmonic function on $\bar{B}$, the first inequality follows. To show the second one, we need to use the positivity of $\alpha$. By (3.2) there is a constant $C>0$ such that

$$
\chi-\frac{1}{C} d d^{c} \rho \in \Gamma_{m}(\alpha),
$$

where $\rho=|z|^{2}-r^{2} \leq 0$. We can choose $C$ such that $|\rho / C| \leq 1 / 2$. Take a function $0 \leq w \leq 1 / 2$ in $\mathcal{A}_{0}$, then it is easy to see that

$$
\int_{E}\left(d d^{c} w\right)^{m} \wedge \alpha^{n-m} \leq \int_{E}\left(\chi+d d^{c}\left(w-\frac{\rho}{C}\right)\right)^{m} \wedge \alpha^{n-m} \leq \operatorname{cap}(E) .
$$

Hence, $\mathbf{c}_{m}(E) \leq 2^{m} \operatorname{cap}(E)$.

Corollary 3.6. Let $u \in \mathcal{A} \cap L^{\infty}(\bar{B})$. Then, $u$ is quasi-continuous with respect to the capacity $\operatorname{cap}(\cdot)$.

Proof. Observe that $v:=u+\varphi$ is $m-\alpha$ subharmonic for some smooth plurisubharmonic function $\varphi$ on $\bar{B}$. Therefore, $v$ is also approximated by a decreasing sequence of smooth $m-\alpha$ subharmonic functions. By the arguments in BedfordTaylor [2] adapted to the case $\omega=e^{G} \alpha$ (see similar arguments in Lemma 9.19), we get that $v$ is quasi-continuous with respect to $\mathbf{c}_{m}(\cdot)$. By Lemma 3.5 the proof is completed.

The next consequence is an inequality between volume and capacity.

Lemma 3.7. Fix $1<\tau<n /(n-m)$. There exists a constant $C(\tau)$ such that for any Borel set $E \subset B$

$$
V_{\alpha}(E) \leq C(\tau)[\operatorname{cap}(E)]^{\tau},
$$

where $V_{\alpha}(E):=\int_{E} \alpha^{n}$. 
The exponent here is optimal because if we take $\alpha=d d^{c}|z|^{2}$, then the explicit formula for $\mathbf{c}_{m}(B(0, s))$ in $B=B(0, r)$ with $0<s<r$, provides an example.

Proof. From [18, Proposition 2.1] we knew that $V_{\alpha}(E) \leq C\left[\mathbf{c}_{m}(E)\right]^{\tau}$ with $\mathbf{c}_{m}(E)$ defined in (3.7). Note that the argument in [18, remains valid for non-Kähler $\alpha$ since the mixed form type inequality used there still holds by stability estimates for the Monge-Ampère equation. Thanks to Lemma 3.5 the proof follows.

3.2. Comparison principles in $\mathcal{A} \cap L^{\infty}(\bar{B})$. For simplicity if $u, v \in \mathcal{A} \cap L^{\infty}(\bar{B})$ we write

$$
u \geq v \quad \text { on } \partial B \text { meaning that } \liminf _{z \rightarrow \partial B}(u-v) \geq 0 .
$$

Lemma 3.8. Let $u, v \in S H_{\chi, m}(\alpha) \cap L^{\infty}(\bar{B})$ be such that $u \geq v$ on $\partial B$. Let $T=\chi_{v_{1}} \wedge \cdots \wedge \chi_{v_{m-1}} \wedge \alpha^{n-m}$ with $v_{i} \in S H_{\chi, m} \cap L^{\infty}(\bar{B})$. Then,

$$
\int_{\{u<v\}} d d^{c} v \wedge T \leq \int_{\{u<v\}} d d^{c} u \wedge T+\int_{\{u<v\}}(v-u) d d^{c} T .
$$

Notice that by the equations (3.4) and (3.5)

$$
\begin{aligned}
d d^{c} T & =d d^{c}\left(e^{(m-n) G} \chi_{v_{1}} \wedge \cdots \wedge \chi_{v_{m-1}} \wedge \omega^{n-m}\right) \\
& =d d^{c}\left(e^{(m-n) G} \chi_{v_{1}} \wedge \cdots \wedge \chi_{v_{m-1}}\right) \wedge \omega^{n-m} .
\end{aligned}
$$

where $\omega=e^{G} \alpha$ is a fixed Kähler form in (3.1).

Proof. By replacing $u$ by $u+\delta$ for $\delta>0$ and then letting $\delta \searrow 0$ we will work with $\{u<v\} \subset \subset K \subset \subset B$, where $K$ is an open set. By the CLN inequality (Lemma 3.3)

$$
\int_{K}\left\|d d^{c} T\right\|<+\infty
$$

By Theorem 3.4. Corollary 3.6, and arguments in [3] we get that

$$
\mathbf{1}_{\{u<v\}} d d^{c} \max \{u, v\} \wedge T=\mathbf{1}_{\{u<v\}} d d^{c} v \wedge T
$$

as two measures. Since $\{u+\varepsilon<v\} \subset \subset K$ for $\varepsilon>0$, Stokes' theorem gives

$$
\begin{aligned}
& \int_{K} d d^{c} \max \{u+\varepsilon, v\} \wedge T \\
& =\int_{\partial K} d^{c} u \wedge T+\int_{K} d^{c} \max \{u+\varepsilon, v\} \wedge d T \\
& =\int_{\partial K} d^{c} u \wedge T+\int_{\partial K} u \wedge d T+\int_{K} \max \{u+\varepsilon, v\} d d^{c} T \\
& =\int_{K} d d^{c} u \wedge T-\int_{K} u d d^{c} T+\int_{K} \max \{u+\varepsilon, v\} d d^{c} T \\
& =\int_{K} d d^{c} u \wedge T+\int_{\{u+\varepsilon<v\} \cap K}(v-u) d d^{c} T+\varepsilon \int_{\{u+\varepsilon \geq v\} \cap K} d d^{c} T .
\end{aligned}
$$


Moreover, by the identity 3.10

$$
\begin{aligned}
& \int_{\{u+\varepsilon<v\}} d d^{c} v \wedge T \\
& =\int_{\{u+\varepsilon<v\}} d d^{c} \max \{u+\varepsilon, v\} \wedge T \\
& =\int_{K} d d^{c} \max \{u+\varepsilon, v\} \wedge T-\int_{\{u+\varepsilon \geq v\} \cap K} d d^{c} \max \{u+\varepsilon, v\} \wedge T \\
& \leq \int_{K} d d^{c} \max \{u+\varepsilon, v\} \wedge T-\int_{\{u+\varepsilon>v\} \cap K} d d^{c} u \wedge T .
\end{aligned}
$$

Thus, it follows that

$$
\begin{aligned}
\int_{\{u+\varepsilon<v\}} d d^{c} v \wedge T \leq & \int_{\{u+\varepsilon \leq v\}} d d^{c} u \wedge T+\int_{\{u+\varepsilon<v\}}(v-u) d d^{c} T \\
& +\varepsilon \int_{K}\left\|d d^{c} T\right\| .
\end{aligned}
$$

Letting $\varepsilon \searrow 0$ we get the desired inequality.

In the Hermitian setting due to the torsion of $\alpha$ and $\chi$, the classical comparison principle no longer holds. However, its weak versions in [17] and 41] are enough for several applications. We state the local counterparts of those.

Let $D_{1}, D_{2}$ be two constants such that on $\bar{B}$,

$$
\begin{aligned}
& -D_{1} \alpha^{2} \leq d d^{c} \alpha \leq D_{1} \alpha^{2}, \quad-D_{1} \alpha^{3} \leq d \alpha \wedge d^{c} \alpha \leq D_{1} \alpha^{3} \\
& -D_{2} \alpha^{2} \leq d d^{c} \chi \leq D_{2} \alpha^{2}, \quad-D_{2} \alpha^{3} \leq d \chi \wedge d^{c} \chi \leq D_{2} \alpha^{3} .
\end{aligned}
$$

Lemma 3.9. Let $u, v \in \mathcal{A} \cap L^{\infty}(\bar{B})$ be such that $u \geq v$ on $\partial B$. Assume that $d=\sup _{\bar{B}}(v-u)>0$. and $D_{1} D_{2} \sup _{\{u<v\}}(v-u) \leq 1$. Then,

$$
\begin{aligned}
& \int_{\{u<v\}}\left(\chi+d d^{c} v\right)^{m} \wedge \alpha^{n-m} \leq \int_{\{u<v\}}\left(\chi+d d^{c} u\right)^{m} \wedge \alpha^{n-m}+ \\
& +C D_{1} D_{2} \sup _{\{u<v\}}(v-u) \sum_{k=0}^{m-1} \int_{\{u<v\}}\left(\chi+d d^{c} u\right)^{k} \wedge \alpha^{n-k} .
\end{aligned}
$$

The constant $C$ depends only on $n, m$.

Proof. We used repeatedly Lemma 3.8 (for $T=\chi_{u}^{k} \wedge \chi_{v}^{l} \wedge \alpha^{n-k-l}, k+l \leq m-1$ ), and bounds in (3.11) to replace $v$ by $u$. Thanks to results in [43, Section 2] the arguments go through for general Hessian operators with respect to the Hermitian metric $\alpha$.

Recall from (3.2) that there exists $0<c_{0} \leq 1$, depending on $\chi, \alpha, \bar{B}$, such that

$$
\chi-c_{0} \alpha \in \Gamma_{m}(\alpha) \text {. }
$$

The weak comparison principle is a crucial tool in pluripotential theory approach to study weak solutions of Hessian type equations [41, 42, 43. We state a local version. 
Lemma 3.10. Let $u, v \in \mathcal{A} \cap L^{\infty}(\bar{B})$ be such that $u \geq v$ on $\partial B$. Assume that $d=\sup _{B}(v-u)>0$. Fix $0<\varepsilon<\min \left\{1 / 2, d /\left(1+2\|v\|_{\left.L^{\infty}\right)}\right\}\right.$. Denote $S(\varepsilon)=$ $\inf _{B}[u-(1-\varepsilon) v]$, and for $s>0$,

$$
U(\varepsilon, s):=\{u<(1-\varepsilon) v+S(\varepsilon)+s\} .
$$

Then, for $0<s<\left(c_{0} \varepsilon\right)^{3} /\left(16 D_{1} D_{2}\right)$,

$$
\int_{U(\varepsilon, s)}\left(\chi+(1-\varepsilon) d d^{c} v\right)^{m} \wedge \alpha^{n-m} \leq\left(1+\frac{C s}{\left(c_{0} \varepsilon\right)^{m}}\right) \int_{U(\varepsilon, s)}\left(\chi+d d^{c} u\right)^{m} \wedge \alpha^{n-m} .
$$

The constant depends on $n, m, D_{1}, D_{2}$.

Proof. We only give here a brief argument as it is very similar to the one of [41, Theorem 2.3]. Set for $0 \leq k \leq m$,

$$
a_{k}:=\int_{U(\varepsilon(s))} \chi_{u}^{k} \wedge \alpha^{n-k}
$$

Then,

$$
\left(c_{0} \varepsilon\right) a_{k} \leq \varepsilon \int_{U(\varepsilon, s)} \chi_{u}^{k} \wedge \chi \wedge \alpha^{n-k-1} \leq \int_{U(\varepsilon, s)} \chi_{u}^{k} \wedge \chi_{(1-\varepsilon) v} \wedge \alpha^{n-k-1} .
$$

By Lemma 3.8

$$
\int_{U(\varepsilon, s)} \chi_{u}^{k} \wedge \chi_{(1-\varepsilon) v} \wedge \alpha^{n-k-1} \leq \int_{U(\varepsilon, s)} \chi_{u}^{k+1} \wedge \alpha^{n-k-1}+R
$$

where $R=\int_{U(\varepsilon, s)}[(1-\varepsilon) v+S(\varepsilon)+s-u] d d^{c}\left(\chi_{u}^{k} \wedge \alpha^{n-k-1}\right)$. It is bounded by

$$
R \leq s D_{1} D_{2}\left(a_{k}+a_{k-1}+a_{k-2}\right),
$$

where we simply understand $a_{k} \equiv 0$ for $k<0$. To be honest, here we used 43 , Lemma 2.3], hence we should multiply the right hand side with a constant $C_{m, n}>0$ depending only on $m, n$. This is no harm as we could adjust the definitions of $D_{1}, D_{2}$.

Thus, for $0<s<\delta:=\frac{\left(c_{0} \varepsilon\right)^{3}}{D_{1} D_{2}},\left(c_{0} \varepsilon\right) a_{k} \leq \delta\left(D_{1} D_{2}\right)\left(a_{k}+a_{k-1}+a_{k-2}\right)+a_{k+1}$. The rest goes in the same way as in [41, Theorem 2.3].

The following result is obvious if potential functions are smooth.

Corollary 3.11. Let $u, v \in \mathcal{A} \cap L^{\infty}(\bar{B})$ be such that $u \geq v$ on $\partial B$. Suppose that $\chi_{u}^{m} \wedge \alpha^{n-m} \leq \chi_{v}^{m} \wedge \alpha^{n-m}$ in $B$. Then, $u \geq v$ on $\bar{B}$.

Proof. It follows from the proof of [41, Corollary 3.4.] with obvious modifications. The reason is that there exists a $C^{2}$ strictly plurisubharmonic function on $\bar{B}$.

We have proved the comparison principle (Lemma 3.10) and volume-capacity inequality (Lemma 3.7). The following uniform a priori estimate is proved in the identically way as [43, Theorem 3.10].

Theorem 3.12. Let $u, v \in \mathcal{A} \cap L^{\infty}(B)$ be such that

$$
\liminf _{z \rightarrow \partial B}(u-v) \geq 0, \quad d:=\sup _{B}(v-u)>0 .
$$


Let us fix the following constants:

$$
\begin{aligned}
& p>n / m, \quad 0<\tau<\frac{p-\frac{n}{m}}{p(n-m)}, \quad \tau^{*}=\frac{(1+m \tau) p}{p-1} ; \\
& 0<\varepsilon<\min \left\{1 / 2, d / 3\left(1+\|v\|_{\infty}\right)\right\} ; \\
& \varepsilon_{0}:=\frac{1}{3} \min \left\{\left(c_{0} \varepsilon\right)^{m}, \frac{\left(c_{0} \varepsilon\right)^{3}}{16 D_{1} D_{2}}\right\} .
\end{aligned}
$$

Suppose that $\left(\chi+d d^{c} u\right)^{m} \wedge \alpha^{n-m}=f \alpha^{n}$ on $B$ with $f \in L^{p}\left(B, \alpha^{n}\right)$. Assume that $v$ is continuous and put

$$
U(\varepsilon, s)=\left\{u<(1-\varepsilon) v+\inf _{B}[u-(1-\varepsilon) v]+s\right\} .
$$

Then, there exists a constant $C=C(\tau, \alpha, B)$ such that for every $0<s<\varepsilon_{0}$,

$$
s \leq C\left(1+\|v\|_{L^{\infty}(B)}\right)\|f\|_{L^{p}(B)}^{\frac{1}{m}}\left[V_{\alpha}(U(\varepsilon, s))\right]^{\frac{\tau}{\tau^{*}}},
$$

where $V_{\alpha}(E)=\int_{E} \alpha^{n}$ for a Borel set $E$.

Notice that from assumptions, the sub-level sets near the infimum point will be non-empty and relatively compact in the ball $B$. The restriction on the class $\mathcal{A}$ will be relaxed later (see Remark 3.19).

3.3. The Dirchlet problems on $\bar{B}$. Consider the Dirichlet problem with the right hand side in $L^{p}(B), p>n / m$. Notice that $n / m$ is the optimal exponent.

$$
\begin{aligned}
& u \in \mathcal{A} \cap C^{0}(\bar{B}), \\
& \left(\chi+d d^{c} u\right)^{m} \wedge \alpha^{n-m}=f \alpha^{n}, \\
& u=\varphi \in C^{0}(\partial B) .
\end{aligned}
$$

Lemma 3.13. Let $f, g$ be non-negative functions in $L^{p}(B), p>n / m$. Let $\varphi, \psi \in$ $C^{0}(\partial B)$. Suppose that $u, v$ are solutions to the corresponding Dirichlet problem (3.13) with the datum $(f, \varphi)$ and $(g, \psi)$. Then,

$$
\|u-v\|_{L^{\infty}(B)} \leq \sup _{\partial B}|\varphi-\psi|+C\|f-g\|_{L^{p}(B)}^{\frac{1}{m}},
$$

where $C$ depends only on $p$ and the diameter of $B$.

Proof. We use an idea in [18, which used the uniform a priori estimate for MongeAmpère equation due to Kołodziej 37. The proof here is similar to 54, Theorem 3.11]. Put $h=|f-g|^{\frac{n}{m}}$ in $B$. It follows that $h \in L^{\frac{p m}{n}}(B)$, where $\frac{p m}{n}>1$. Moreover,

$$
\|h\|_{L^{\frac{p m}{n}}(B)}^{\frac{1}{n}}=\|f-g\|_{L^{p}(B)}^{\frac{1}{m}} .
$$

By a theorem in [37, there exists $\rho \in P S H(B) \cap C^{0}(\bar{B})$ solving

$$
\left(d d^{c} \rho\right)^{n}=h \alpha^{n}, \quad \rho_{\left.\right|_{\partial B}}=0 .
$$

We also have

$$
\|\rho\|_{L^{\infty}} \leq C\|h\|_{L^{\frac{p m}{n}}(B)}^{\frac{1}{n}}=C\|f-g\|_{L^{p}(B)}^{\frac{1}{m}},
$$

where $C=C(m, n, p, B, \alpha)$ a uniform constant. Furthermore, by the mixed-form inequality,

$$
\left(d d^{c} \rho\right)^{m} \wedge \alpha^{n-m} \geq h^{\frac{m}{n}} \alpha^{n}=|f-g| \alpha^{n} .
$$


Therefore,

$$
\begin{aligned}
{\left[\chi_{u}+d d^{c} \rho\right]^{m} \wedge \alpha^{n-m} } & \geq \chi_{u}^{m} \wedge \alpha^{n-m}+\left(d d^{c} \rho\right)^{m} \wedge \alpha^{n-m} \\
& \geq f \alpha^{n}+|f-g| \alpha^{n} \\
& \geq g \alpha^{n} .
\end{aligned}
$$

Since $\rho \leq 0$ in $\bar{B}$, it follows from the domination principle (Corollary 3.11) that $u+\rho \leq v+\sup _{\partial B}|u-v|$. Hence,

$$
u-v \leq-\rho+\sup _{\partial B}|u-v| \leq \sup _{\partial B}|u-v|+C\|f-g\|_{L^{p}(B)}^{\frac{1}{m}} .
$$

Similarly, $v-u \leq \sup _{\partial B}|u-v|+C\|f-g\|_{L^{p}(B)}^{\frac{1}{m}}$. Thus, the theorem follows.

We also need another stability estimate for solutions whose Hessian operators are in $L^{p}, p>n / m$.

Lemma 3.14. Under the assumptions of Lemma 3.13 there exist a uniform constant $C=C\left(p, m, n,\|f\|_{p},\|g\|_{p}\right)$ and a constant $a=a(p, m, n)>0$ such that

$$
\|u-v\|_{L^{\infty}(B)} \leq \sup _{\partial B}|\varphi-\psi|+C\|u-v\|_{L^{1}(B)}^{a} .
$$

Proof. Having Theorem 3.12 we can repeat the proof of [43, Theorem 3.11] two times, one for the pair $u+\sup _{\partial B}|\varphi-\psi|$ and $v$ and another for the pair $v+$ $\sup _{\partial B}|\varphi-\psi|$ and $u$.

We get from the existence of smooth solutions (Theorem 1.1) and stability estimates (Lemma 3.13) existence of weak solutions.

Theorem 3.15. Let $0 \leq f \in L^{p}(B)$ with $p>n / m$. Then, there exists a unique solution to the Dirichlet problem (3.13).

The last ingredient to prove the approximation property for $(\chi, m)-\alpha$-subharmonic functions is the existence of smooth solutions for a Hessian type equation.

Lemma 3.16. Let $H$ be a smooth function on $\bar{B}$ and $\varphi \in C^{\infty}(\partial B)$. Then, there exists a unique $u \in S H_{\chi, m}(\alpha) \cap C^{\infty}(\bar{B})$ solving the Hessian equation

$$
\begin{array}{r}
\left(\chi+d d^{c} u\right)^{m} \wedge \alpha^{n-m}=e^{u+H} \alpha^{n}, \\
u=\varphi \quad \text { on } \partial B .
\end{array}
$$

Proof. The right hand side depends also on $u$ but with the right sign. We solve the equation by the continuity method as in the proof of Theorem 1.3, provided second order apriori estimates. The $C^{0}$-estimate easily follows by considering the maximum point and the minimum point of the solution. So does the $C^{1}$-estimate on the boundary. The proof of $C^{1}$-estimate at an interior point will be affected at equations (7.8) and (7.9) in Section 7 . The extra terms appear in these equations are $O\left(|\nabla u|^{2}\right)$. So this will not affect the conclusion of the inequality (7.10). Therefore, we will get $C^{1}$-estimate. The $C^{2}$-estimate at an interior point goes through as in Section 8, as it is explained in [43, Lemma 3.18]). For the other $C^{2}$-estimates at a boundary point, the equation (8.12) contains a bounded term $O(|\nabla u|)$ by the $C^{1}$-estimate. Therefore, the equality (8.13) will still hold and we get the desired estimates. 
Lemma 3.17. Let $0 \leq f \in L^{p}(B), p>n / m$, and $\varphi \in C^{0}(\partial B)$. Let $\left\{f_{j}\right\}_{j \geq 1}$ be smooth and positive functions on $\bar{B}$, converging in $L^{p}(\bar{B})$ to $f$ as $j \rightarrow+\infty$. Let $\varphi_{j} \in C^{\infty}(\partial B)$ converge uniformly to $\varphi$. Assume that

$$
\begin{array}{r}
\chi_{u_{j}}^{m} \wedge \alpha^{n-m}=e^{u_{j}} f_{j} \alpha^{n}, \\
u_{j}=\varphi_{j} \quad \text { on } \partial B .
\end{array}
$$

Then, $u_{j}$ converges uniformly to $u \in \mathcal{A} \cap C^{0}(\bar{B})$, which is the unique solution in $\mathcal{A} \cap C^{0}(\bar{B})$ of

$$
\begin{array}{r}
\chi_{u}^{m} \wedge \alpha^{n-m}=e^{u} f \alpha^{n}, \\
u=\varphi \quad \text { on } \partial B .
\end{array}
$$

Proof. Observe that $u_{j}$ is uniformly bounded above. It follows that the right hand side of equations are uniformly bounded in $L^{p}$. Applying Lemma 3.13 for $\psi=0$ and $g=0$, this gives the uniform bound for $u_{j}$. Then, by compactness of the sequence $u_{j}$ in $L^{1}$ and Lemma 3.14 we get a continuous solution by passing to the limit. The uniqueness follows as in [55, Lemma 2.3].

3.4. Approximation property on $\bar{B}$. We have all ingredients which are needed to prove the main theorem of this section. By using results of Pliś [59, Harvey Lawson - Pliś [34, Theorem 6.1] also proved this theorem in the case $\chi \equiv 0$ and $\alpha$ being Kähler.

Theorem 3.18. Let $u$ be $(\chi, m)-\alpha$-subharmonic in a neighborhood of $\bar{B}$. Then, there exists a sequence of smooth functions $u_{j} \in S H_{\chi, m}(\alpha) \cap C^{\infty}(\bar{B})$ such that $u_{j}$ decreases to $u$ point-wise in $B$ as $j$ goes to $+\infty$.

Proof. We follow closely the proof of [43, Lemma 3.20], which in turns uses the scheme introduced by Berman [4] and Eyssidieux-Guedj-Zeriahi [23] (see also LuNguyen [51).

By positivity assumption on $\chi \in \Gamma_{m}(\alpha)$ for every $z \in \bar{B}$ we have that $j \in$ $S H_{\chi, m}(\alpha)$ for any constant $j$. As $\max \{u,-j\}$ belongs to $S H_{\chi, m}(\alpha)$, we may assume that $u$ is bounded. Since $u$ is upper semicontinuous on $\bar{B}$, there exists a sequence of smooth functions $\phi_{j}$ decreasing to $u$ on $\bar{B}$. Fix such an $h:=\phi_{j}$. Consider the envelope

$$
\tilde{h}:=\sup \left\{v \in S H_{\chi, m}(\alpha) \cap L^{\infty}(B): v \leq h\right\} .
$$

Then, $\tilde{h} \in S H_{\chi, m}(\alpha)$ and $u \leq \tilde{h} \leq h$. Therefore, if $\tilde{h} \in \mathcal{A}$, i.e. it has the approximation property, then so does $u$ by letting $h=\phi_{j} \searrow u$. We shall prove that the function $\tilde{h}$ can be approximated uniformly, and then the lemma will follow.

Since $h \in C^{\infty}(\bar{B})$, we can write $\chi_{h}^{m} \wedge \alpha^{n-m}=F \alpha^{n}$ with $F$ being a smooth function on $\bar{B}$. Let us denote $F_{*}=\max \{F, 0\}$. We choose a sequence of smoothly non-negative functions $F_{j}$ decreasing uniformly to $F_{*}$ as $j \rightarrow \infty$. Fix such a $\tilde{F}:=$ $F_{j} \geq F_{*}$. By Lemma 3.16 we solve for $0<\varepsilon \leq 1$,

$$
\begin{array}{r}
\chi_{\tilde{w}_{\varepsilon}}^{m} \wedge \alpha^{n-m}=e^{\frac{1}{\varepsilon}\left(\tilde{w}_{\varepsilon}-h\right)}[\tilde{F}+\varepsilon] \alpha^{n}, \\
\tilde{w}_{\varepsilon}=h \quad \text { on } \partial B .
\end{array}
$$

By maximum principle, $\tilde{w}_{\varepsilon} \leq h$ and $\tilde{w}_{\varepsilon}$ is increasing as $\varepsilon$ decreases to 0 . Keep $\varepsilon$ fixed, and take limit on both sides for $\tilde{F}=F_{j} \rightarrow F_{*}$, i.e. letting $j \rightarrow \infty$, we get 
from Lemma 3.17

$$
\begin{array}{r}
\chi_{w_{\varepsilon}}^{m} \wedge \alpha^{n-m}=e^{\frac{1}{\varepsilon}\left(w_{\varepsilon}-h\right)}\left[F_{*}+\varepsilon\right] \alpha^{n}, \\
w_{\varepsilon}=h \text { on } \partial B .
\end{array}
$$

Here $\tilde{w}_{\varepsilon}$ uniformly increases to $w_{\varepsilon}$. Thus, $w_{\varepsilon} \in \mathcal{A} \cap C^{0}(\bar{B})$ and $w_{\varepsilon}$ is increasing as $\varepsilon$ decreases to 0 . Since $w_{\varepsilon} \leq h$, the right hand side is uniformly bounded in $L^{\infty}(\bar{B})$. The monotone sequence $w_{\varepsilon}$, bounded above by $h$, is a Cauchy sequence in $L^{1}(B)$. By Lemma 3.14 this sequence is also Cauchy in the uniform norm in $\bar{B}$. So, $w_{\varepsilon}$ uniformly increases to $w$ which satisfies

$$
\begin{array}{r}
\chi_{w}^{m} \wedge \alpha^{n-m} \leq \mathbf{1}_{\{w=h\}} F_{*} \alpha^{n}, \\
w=h \quad \text { on } \partial B .
\end{array}
$$

In particular, $w \in \mathcal{A} \cap C^{0}(\bar{B})$. Now, we claim that $w=\tilde{h}$. The inequality $w \leq \tilde{h}$ is clear. One needs to verify that $w \geq \tilde{h}$ on $\{w<h\}$. Take a candidate $v$ in the envelope (3.14), i.e, $v \leq h$. Observe that $\chi_{w}^{m} \wedge \alpha^{n-m}=0$ on $\{w<v\} \subset\{w<h\}$. By Corollary 3.11 it follows that $w$ is maximal on $\{w<h\}$. Thus, the set $\{w<v\}$ is empty, i.e., $w \geq v$. Since $v$ is arbitrary, so $w \geq \tilde{h}$. The claim follows and so does the theorem.

Remark 3.19. (a) In the proof we only used the wedge product for continuous potential, so Theorem 3.18 holds for a general Hermitian metric $\alpha$. In this case one should use a counterpart of [43, Theorem 2.16] instead of Corollary 3.11] in the last argument.

(b) An immediate consequence is that the class $\mathcal{A}$ coincides with $S H_{\chi, m}(\alpha)$.

Thanks to the quasi-continuity and approximation property of $(\chi, m)-\alpha$ - subharnonic functions we get an inequality similar to the one for plurisubharmonic functions in Cegrell-Kołoldziej [11.

Proposition 3.20. Let $u, v \in S H_{\chi, m}(\alpha) \cap L^{\infty}(B)$. Let $\mu$ be a positive measure such that $\chi_{u}^{m} \wedge \alpha^{n-m} \geq \mu$ and $\chi_{v}^{m} \wedge \alpha^{n-m} \geq \mu$. Then

$$
\left(\chi+d d^{c} \max \{u, v\}\right)^{m} \wedge \alpha^{n-m} \geq \mu .
$$

Proof. It is readily adaptable from [11, Theorem 1] with an obvious change of notations.

\section{The Dirichlet Problem}

On the complex manifold $M=\bar{M} \backslash \partial M$ we define the class $S H_{\chi, m}(\alpha, M)$ in local coordinates. One main difference is that for an arbitrary real $(1,1)$-form $\chi$ on $M$, there are plenty of local $(\chi, m)-\alpha$-subharmonic functions on each local chart. However, the global class $S H_{\chi, m}(\alpha, M)$ may be empty, e.g. for negative $\chi$. Thus, the existence of a subsolution will guarantee that $S H_{\chi, m}(\alpha)$ is non empty.

In this section we shall study weak solutions to the Dirichlet problem for the complex Hessian type equation. As we pointed out in Section 3.1 the assumption $\alpha$ is locally conformal Kähler metric on $M$ is needed to develop potential theory for bounded functions.

Fix the continuous right hand side density $0 \leq f \in C^{0}(\bar{M})$ and a continuous boundary data $\varphi \in C^{0}(\partial M)$. Let us denote

$$
\mu:=f \alpha^{n} .
$$


We wish to solve the Dirichlet problem:

$$
\begin{aligned}
& w \in S H_{\chi, m}(\alpha) \cap C^{0}(\bar{M}), \\
& \left(\chi+d d^{c} w\right)^{m} \wedge \alpha^{n-m}=\mu, \\
& w=\varphi \text { on } \partial M .
\end{aligned}
$$

The $C^{2}$ subsolution $\rho$ to the equation (4.1) satisfies:

$$
\chi_{\rho}:=\chi+d d^{c} \rho \in \Gamma_{m}(\alpha),
$$

and

$$
\left(\chi+d d^{c} \rho\right)^{m} \wedge \alpha^{n-m} \geq \mu, \quad \rho=\varphi \quad \text { on } \partial M .
$$

By replacing $\chi$ by $\chi_{\rho}$ and $u$ by $u-\rho$ we can reduce the problem to the case of zero boundary data and $\chi \in \Gamma_{m}(\alpha)$ as follows:

$$
\begin{aligned}
& w \in S H_{\chi, m}(\alpha) \cap C^{0}(\bar{M}), \\
& \left(\chi+d d^{c} w\right)^{m} \wedge \alpha^{n-m}=\mu, \\
& w=0 \quad \text { on } \partial M .
\end{aligned}
$$

Then 0 is the subsolution to the equation (4.3), and there exists $0<c_{0} \leq 1$ such that

$$
\chi-c_{0} \alpha \in \Gamma_{m}(\alpha) .
$$

4.1. Envelope of continuous subsolutions. By assumption (4.2) the set

$$
\mathcal{S}=\left\{v \in S H_{\chi, m}(\alpha) \cap C^{0}(\bar{M}): \chi_{v}^{m} \wedge \alpha^{n-m} \geq \mu, v_{\left.\right|_{\partial M}} \leq 0\right\}
$$

is not empty. Hence, we define the envelope

$$
u_{0}(z):=\sup _{v \in \mathcal{S}} v(z) .
$$

One expects that it will be a solution to the continuous Dirichlet problem.

Theorem 4.1. If $u_{0}$ is continuous, then it solves the Dirichlet problem (4.1).

Proof. We first have $u_{0} \in S$ by Proposition 2.6-(b) and Proposition 3.20. In particular,

$$
\left(\chi+d d^{c} u_{0}\right)^{m} \wedge \alpha^{n-m} \geq \mu .
$$

It remains to show that $\chi_{u_{0}}^{m} \wedge \alpha^{n-m}=\mu$. Fix a small ball $B \subset M$ and find $w \in S H_{\chi, m}(\alpha) \cap C^{0}(\bar{B})$ solving $w=u_{0}$ on $\partial B$ and

$$
\left(\chi+d d^{c} w\right)^{m} \wedge \alpha^{n-m}=\mu \quad \text { in } B .
$$

Hence, $w \geq u_{0}$ in $\bar{B}$. Consider the lift $\tilde{u} \in \mathcal{S}$ of $u_{0}$ with respect to this ball defined by

$$
\tilde{u}=\left\{\begin{array}{l}
\max \left\{w, u_{0}\right\} \quad \text { on } B, \\
u_{0} \text { on } \bar{M} \backslash B .
\end{array}\right.
$$

Thus, we have $\tilde{u} \in S$ and $u_{0} \leq \tilde{u}$ in $B$. On the other hand by the definition of $u_{0}$ we have $\tilde{u} \leq u_{0}$. Thus, $u_{0}=\tilde{u}$ in $B$, which means $\chi_{u_{0}}^{m} \wedge \alpha^{n-m}=\mu$. This holds for any ball, so the theorem follows.

Remark 4.2. For continuous $(\chi, m)-\alpha$-subharmonic functions the wedge product is always well-defined. Theorem 4.1 is valid for a general Hermitian metric $\alpha$. The remaining issue is to verify the continuity of the envelope $u_{0}$. So far we could not do this for a general Hermitian metric $\alpha$. 
Remark 4.3. Let us consider $m=n$ and $f \equiv 0$ in connection with the geodesic equation studied notably by Semmes [60, Donaldson [21, Chen [10] and Blocki 7. It follows from the comparison principle (an extension of Lemma 3.10 for $M$ in the place of $B$ ), that there exists at most one continuous solution to the equation. Guan and $\mathrm{Li}$ [28] have extended the gradient estimate in [6] to this case. Hence, we can get a continuous solution to the homogeneous equation by a compactness argument. This solution is maximal on $M$, thus equal to $u_{0}$. Thus, we get the unique solution even in the case the background metric is only Hermitian.

4.2. Envelope of bounded subsolutions. In this section we shall prove Theorem 1.3. where $\alpha$ is locally conformal Kähler. First we enlarge the class $\mathcal{S}$ above,

$$
\hat{\mathcal{S}}:=\left\{v \in S H_{\chi, m}(\alpha) \cap L^{\infty}(\bar{M}): \chi_{v}^{m} \wedge \alpha^{n-m} \geq \mu, v_{\left.\right|_{\partial M} ^{*}}^{*} \leq 0\right\} .
$$

The locally conformal Kähler assumption of $\alpha$ allows us to use potential theory which has been developed in Section 3 for bounded $(\chi, m)-\alpha$-subharmonic functions. Set

$$
u(z):=\sup _{v \in \hat{\mathcal{S}}} v(z)
$$

It follows from Proposition 2.6 (b) and Proposition 3.20 that $u^{*} \in \hat{\mathcal{S}}$. Hence, $u=u^{*}$. Let us solve the linear PDE

$$
\begin{array}{r}
\left(\chi+d d^{c} \rho_{1}\right) \wedge \alpha^{n-1}=0, \\
\rho_{1}=0 \quad \text { on } \partial M .
\end{array}
$$

Therefore, $0 \leq u \leq \rho_{1}$. It implies that $u=0$ and it is continuous on $\partial M$.

Remark 4.4. It is obvious that $u_{0} \leq u$. If we can show that $u$ is a continuous on $M$, then $u \in \mathcal{S}$ automatically. Then, $u_{0}=u$ is indeed continuous.

In what follows, we shall prove that $u$ is a solution to the (bounded) Dirichlet problem, and then we will prove its regularity by using the a priori estimate (Theorem 3.12).

Lemma 4.5 (lift). Let $v \in \hat{\mathcal{S}}$. Let $B \subset M$ be a small ball. There exists $\tilde{v} \in \hat{\mathcal{S}}$ such that $v \leq \tilde{v}$ and $\chi_{\tilde{v}}^{m} \wedge \alpha^{n-m}=\mu$ in $B$.

Proof. Choose $C^{0}(\partial B) \ni \phi_{j} \searrow v$ on $\partial B$ and solve the Dirichlet problem

$$
\left\{\begin{array}{l}
v_{j} \in S H_{\chi, m}(\alpha) \cap C^{0}(\bar{B}), \\
\left(\chi+d d^{c} v_{j}\right)^{m} \wedge \alpha^{n-m}=\mu, \\
v_{j}=\phi_{j} \text { on } \partial B .
\end{array}\right.
$$

It follows from Corollary 3.11 that $v_{j} \searrow w \in S H_{\chi, m}(\alpha, B)$. Hence, Theorem 3.4 gives that

$$
\left(\chi+d d^{c} w\right)^{m} \wedge \alpha^{n-m}=\mu .
$$

Furthermore, $\lim \sup _{z \rightarrow \zeta \in \partial B} w(z) \leq v(\zeta)$. By the domination principle (Corollary 3.11) we have $v_{j} \geq v$ on $B$. Thus, $w \geq v$ on $B$. Define

$$
\tilde{v}=\left\{\begin{array}{l}
\max \{w, v\} \quad \text { on } B \\
v \quad \text { on } \bar{M} \backslash B .
\end{array}\right.
$$

Then, $\tilde{v}$ is the function we are looking for.

Lemma 4.6. $u \in S H_{\chi, m}(\alpha) \cap L^{\infty}(M) \cap C^{0}(\partial M)$ and $\chi_{u}^{m} \wedge \alpha^{n-m}=\mu$. 
Proof. It only remains to show that $\chi_{u}^{m} \wedge \alpha^{n-m}=\mu$. Fix a small ball $B \subset M$ and consider the lift $\tilde{u} \in \hat{\mathcal{S}}$ of $u$ with respect to this ball. Then, $u \leq \tilde{u}$ in $B$. On the other hand by the definition of $u$ we have $\tilde{u} \leq u$. Thus, $u=\tilde{u}$ in $B$. Since $B$ is arbitrary, $\chi_{u}^{m} \wedge \alpha^{n-m}=\mu$ on $M$.

We shall prove the most technical part.

Lemma 4.7. $u$ is continuous on $\bar{M}$.

By Lemma 4.6, the function $u$ satisfies the (bounded) Dirichlet problem:

$$
\begin{aligned}
& w \in S H_{\chi, m}(\alpha) \cap L^{\infty}(M), \\
& \left(\chi+d d^{c} w\right) \wedge \alpha^{n}=\mu, \\
& \lim _{z \rightarrow \zeta} w(z)=0 \quad \text { for every } \zeta \in \partial M .
\end{aligned}
$$

Proof of Lemma 4.7. We follow closely [38, Section 2.4]. We argue by contradiction. Suppose $u$ is not continuous, then the discontinuity of $u$ occurs at an interior point of $M$. Hence

$$
d=\sup _{\bar{M}}\left(u-u_{*}\right)>0
$$

where $u_{*}(z)=\lim _{\epsilon \rightarrow 0} \inf _{w \in B(z, \epsilon)} u(w)$ is lower regularisation of $u$. Consider the closed nonempty set

$$
F=\left\{u-u_{*}=d\right\} \subset \subset M .
$$

One remark is that $u_{\mid F}$ is continuous on $F$. Therefore, we may choose a point $x_{0} \in F$ such that

$$
u\left(x_{0}\right)=\min _{F} u .
$$

Choose a local coordinate chart about $x_{0}$, relatively compact in $M$, which is isomorphic to a small ball $B:=B(0, r) \subset \mathbb{C}^{n}$ with origin at $z\left(x_{0}\right)=0$ and of small radius. Since $\chi \in \Gamma_{m}(\alpha)$, there exists $\delta>0$ such that

$$
\gamma(z):=\chi(z)-\delta d d^{c}|z|^{2} \in \Gamma_{m}(\alpha)
$$

for every $z \in \bar{B}$. Set

$$
v:=u+\delta|z|^{2} \in S H_{\gamma, m}(\alpha) .
$$

Since $u \geq 0$ on $M, v_{*}(0)=u_{*}(0) \geq 0$. Hence, we have $v \in L^{\infty}(\bar{B})$, which solves

$$
\left(\gamma+d d^{c} v\right)^{m} \wedge \alpha^{n-m}=\mu .
$$

We also find that

$$
\sup _{\bar{B}}\left(v-v_{*}\right)=\sup _{\bar{B}}\left(u-u_{*}\right)=u(0)-u_{*}(0)=d .
$$

Let us consider the sublevel sets, for $0<s<d$,

$$
E(s)=\left\{u_{*} \leq u-d+s\right\} \cap \bar{B} .
$$

It's clear that $E(s)$ is closed and by our assumption $0 \in E(s)$. Furthermore,

$$
E(s) \searrow E(0)=\left\{u_{*}=u-d\right\} \cap \overline{B(0, r)} \ni 0 .
$$

Let us denote

$$
\tau(s)=u(0)-\inf _{E(s)} u(z) .
$$

Since $E(s)$ is decreasing, it follows that $\tau(s)$ decreasing as $s \searrow 0$. Moreover, $\tau(s)$ is bounded for $0 \leq s \leq d$. We also need the following fact. 
Claim 4.8. $\lim _{s \rightarrow 0} \tau(s)=0$.

Proof of Claim 4.8. It is easy to see that $\liminf _{s \rightarrow 0} \tau(s) \geq \tau(0)=0$. It is enough to show that $\limsup _{s \rightarrow 0} \tau(s) \leq 0$. Suppose that it is not true, i.e.,

$$
\limsup _{s \rightarrow 0} \tau(s)=2 \epsilon>0
$$

for some $\epsilon>0$. Then, there exists a sequence $s_{j} \rightarrow 0$ such that $\tau\left(s_{j}\right)>\epsilon$ for every integer $j>0$. It means that

$$
\inf _{E\left(s_{j}\right)} u<u(0)-\epsilon .
$$

Therefore, there is a sequence $\left\{z_{j}\right\}_{j \geq 1} \subset E\left(s_{j}\right)$ satisfying $u\left(z_{j}\right)<u(0)-\epsilon$. Since any limit point $z$ of $\left\{z_{j}\right\}_{j \geq 1}$ belongs to $E(0), u(z) \geq u(0)$. Hence,

$$
\limsup _{j \rightarrow \infty} u\left(z_{j}\right) \leq u(0)-\epsilon \leq u(z)-\epsilon
$$

The upper semicontinuity of $-u_{*}$ gives

$$
\limsup _{j \rightarrow+\infty}\left[-u_{*}\left(z_{j}\right)\right] \leq-u_{*}(z) .
$$

Hence, $d=\limsup _{j \rightarrow+\infty}\left[u\left(z_{j}\right)-u_{*}\left(z_{j}\right)\right] \leq u(z)-\epsilon-u_{*}(z)=d-\epsilon$. This is not possible and the claim follows.

Take $B^{\prime}=B\left(0, r^{\prime}\right)$ with a bit larger $r^{\prime}>r$. By the approximation property in a small ball (Theorem 3.18), one can find a sequence

$$
S H_{\gamma, m}(\alpha) \cap C^{\infty}\left(B^{\prime}\right) \ni v_{j} \searrow v=u+\delta|z|^{2} \quad \text { in } B^{\prime} .
$$

Let us fix this sequence from now on. If there is no otherwise indication then $v$ and $v_{j}$ 's are these functions. The following result is a variation of the Hartogs lemma (Lemma 9.14).

Lemma 4.9. Let $K \subset \bar{B}$ be a compact set and $c \geq 1$ a constant. Assume that for some $t>0$,

$$
v<c v_{*}+t \quad \text { on } K \text {. }
$$

Then

$$
v_{j}<c v+t \text { on } K
$$

for $j>j_{0}$ with a fixed $j_{0}>0$ depending only on $K, t$.

Proof of Lemma 4.9. Let $z_{0} \in K$. It follows from the assumption that $z_{0} \in\{v-$ $\left.c v_{*}<t\right\}$ which is an open set by the upper semicontinuity of $v-c v_{*}$. Thus, $z_{0} \in\left\{v-c v_{*}<t^{\prime}\right\}$ for some $0<t^{\prime}<t$. Hence, $v\left(z_{0}\right)-c v_{*}\left(z_{0}\right)<t^{\prime}$, i.e., by definition

$$
\lim _{\epsilon^{\prime} \rightarrow 0}\left(\sup _{B\left(z_{0}, 2 \epsilon^{\prime}\right)} v-c \inf _{B\left(z_{0}, 2 \epsilon^{\prime}\right)} v\right)<t^{\prime} .
$$

Therefore, for $0<t_{1}=\frac{t-t^{\prime}}{2}$, there exists $\epsilon^{\prime}=\epsilon^{\prime}\left(t_{1}, z_{0}\right)>0$ such that

$$
B\left(z_{0}, 2 \epsilon^{\prime}\right) \subset\left\{v<v_{*}+t\right\},
$$

and $\sup _{B\left(z_{0}, 2 \epsilon^{\prime}\right)} v-c \inf _{B\left(z_{0}, 2 \epsilon^{\prime}\right)} v \leq t^{\prime}+t_{1}$. It implies that

$$
\sup _{\bar{B}\left(z_{0}, \epsilon^{\prime}\right)} v \leq c v+t^{\prime}+t_{1} \quad \text { on } \bar{B}\left(z_{0}, \epsilon^{\prime}\right) \text {. }
$$


By Hartogs' lemma for $(\gamma, 1)-\alpha$-subharmonic functions (Corollary 9.15),

$$
v_{j} \leq \sup _{\bar{B}\left(z_{0}, \epsilon^{\prime}\right)} v+t_{1}<c v+t^{\prime}+2 t_{1}=c v+t .
$$

for $j \geq j\left(t_{1}, z_{0}, \epsilon^{\prime}\right)$. Because $K$ is compact it is covered by a finite many balls $B\left(z_{j}, \epsilon_{j}^{\prime}\right)$. Thus, the proof follows.

We wish to apply Theorem 3.12 for the function $v$ and its approximants $v_{j}^{\prime} s$ defined in (4.8) to get a contradiction. Therefore, we need to study the value of $v$ and $v_{j}$ 's on the boundary $\partial B$. More precisely, we are going to show that there exists $c>1, a>0$ and $s_{0}$, which are independent of $j$, such that

$$
\left\{c v+d-a+s<v_{j}\right\}
$$

is non-empty and relatively compact in $B=B(0, r)$ for every $0<s<s_{0}$. For this purpose we need to analyse the value of the function $c v-v_{j}$ on the boundary $S(0, r)$ of $B(0, r)$, with the help of Lemma 4.9.

Take two parameters $c>1$ and $0<a<d$ which are determined later. We need to estimate

$$
c v+d-a-v_{j}
$$

on $S(0, r)$. Recall that $v=u+\delta|z|^{2}$ and

$$
\begin{aligned}
E(s) & =\left\{u_{*} \leq u-d+s\right\} \cap \overline{B(0, r)} \\
& =\left\{v_{*} \leq v-d+s\right\} \cap \overline{B(0, r)} .
\end{aligned}
$$

We consider two cases:

Case 1: $z \in S(0, r) \cap E(a)$. We have

$$
\begin{aligned}
v_{*}(z) & =u_{*}(z)+\delta r^{2} \\
& \geq u(z)-d+\delta r^{2} \\
& =(u(z)-u(0))+(u(0)-d)+\delta r^{2} .
\end{aligned}
$$

As $0 \in E(a)$, we have $\tau(a) \geq u(0)-u(z)$. Combining with $u(0)-u_{*}(0)=d$, we get that

$$
v_{*}(z) \geq v_{*}(0)-\tau(a)+\delta r^{2} .
$$

Note that $r>0$ (small) is already fixed. It implies that, for $c>1$,

$$
v(z) \leq v_{*}(z)+d<c v_{*}(z)+d-(c-1)\left[v_{*}(0)+\delta r^{2}-\tau(a)\right] .
$$

Since $v-c v_{*}$ is upper semicontinuous,

$$
v<c v_{*}+d-(c-1)\left[v_{*}(0)+\delta r^{2}-\tau(a)\right]
$$

on the closure of a neighbourhood $V$ of $S(0, r) \cap E(a)$. Applying Lemma 4.9 for the compact set $\bar{V} \cap \bar{B}$ and

$$
t:=d-(c-1)\left[v_{*}(0)+\delta r^{2}-\tau(a)\right]>0,
$$

we get

$$
v_{j}<c v+d-(c-1)\left[v_{*}(0)+\delta r^{2}-\tau(a)\right] \quad \text { on } \bar{V} \cap \bar{B},
$$

if $j>j_{1}(V)$.

Case 2: $z \in S(0, r) \backslash V$. Since $E(a) \cap(S(0, r) \backslash V)=\emptyset$, the inequality

$$
v<v_{*}+d-a
$$


holds on $S(0, r) \backslash V$. Applying Lemma 4.9 again, we get

$$
v_{j}<v+d-a<c v+d-a \text { on } S(0, r) \backslash V
$$

for $j>j_{2}(V)$. Thus, it follows from (4.11) and (4.12) that

$$
v_{j}<c v+d-\min \left\{a,(c-1)\left[v_{*}(0)+\delta r^{2}-\tau(a)\right]\right\}
$$

on $S(0, r)$ for $j>\max \left\{j_{1}, j_{2}\right\}$.

Next, if there exists $c>1$ such that for $0<s_{0}<a$,

$$
(c-1) v_{*}(0)<a-s_{0}
$$

then $c v_{*}(0)+d-\left(a-s_{0}\right)<v(0) \leq v_{j}(0)$. It follows that the set $\left\{c v+d-a+s<v_{j}\right\}$ is non-empty for $0<s<s_{0}$.

According to Claim 4.8, (4.10), (4.13) and (4.14) we need to choose $0<a<d$, $c>1$ and $0<s_{0}<a$, in this order, such that

$$
\begin{aligned}
& \tau(a) \leq \frac{\delta r^{2}}{2} \\
& d-(c-1)\left[v_{*}(0)+\delta r^{2}-\tau(a)\right]>0 ; \\
& (c-1) v_{*}(0)<a<(c-1)\left(v_{*}(0)+\frac{\delta r^{2}}{2}\right) ; \\
& s_{0}=\frac{a-(c-1) v_{*}(0)}{2}>0 .
\end{aligned}
$$

This is always possible. Thus, we get relatively compact subsets that satisfy (4.9).

Now we can apply Theorem 3.12 to get that a contradiction. In fact, we have for $w_{j}:=v_{j} / c$ and $0<s<s_{0}$,

$$
\left\{c v+d-a+s<v_{j}\right\}=\left\{v+(d-a+s) / c<w_{j}\right\} \subset \subset B .
$$

It follows that

$$
d_{j}:=\sup _{B}\left(w_{j}-v\right) \geq \frac{d-a+s_{0}}{c}>0 .
$$

We denote for $0<s<\varepsilon_{0}<\varepsilon$ (as in Theorem 3.12),

$$
U_{j}(\varepsilon, s):=\left\{v<(1-\varepsilon) w_{j}+\inf _{\Omega}\left[v-(1-\varepsilon) w_{j}\right]+s\right\} .
$$

Notice that $\varepsilon_{0}$ depends only on $d, a, s_{0}$. Hence, applying Theorem 3.12 for $v$ in (4.6) and $\gamma$ in (4.5), we get that for $0<s<\varepsilon_{0}$,

$$
s \leq C\left(1+\|v\|_{L^{\infty}}\right)\|f\|_{L^{p}}^{\frac{1}{m}}\left[V_{\alpha}\left(U_{j}(\varepsilon, s)\right)\right],
$$

where $V_{\alpha}\left(U_{j}(\varepsilon, s)\right)=\int_{U_{j}(\varepsilon, s)} \alpha^{n}$. Furthermore, for such a fixed $s>0$,

$$
U_{j}(\varepsilon, s) \subset\left\{v<w_{j}-d_{j}+\varepsilon\left\|w_{j}\right\|_{L^{\infty}}+s\right\} \subset\left\{v<v_{j}\right\} .
$$

Since $V_{\alpha}\left(\left\{v<v_{j}\right\}\right) \rightarrow 0$ as $j \rightarrow+\infty$, we get the contradiction. The proof of Lemma 4.7 is finished. 
4.3. Some applications. The first application is the mixed type inequality for Hessian operators with the Hermitian form. When both $\chi$ and $\omega$ are Kähler metrics the inequality is proved by Dinew and $\mathrm{Lu} 20$. Since the inequality is local, we state it for a small Euclidean ball $B$ in $\mathbb{C}^{n}$.

Proposition 4.10. Let $f, g \in L^{p}(B), p>n / m$. Suppose that $u, v \in S H_{\chi, m}(\alpha) \cap$ $C^{0}(\bar{B})$ satisfy

$$
\chi_{u}^{m} \wedge \alpha^{n-m}=f \alpha^{n}, \quad \chi_{v}^{m} \wedge \omega^{n-m}=g \alpha^{n} .
$$

Then, for any $0 \leq k \leq m$,

$$
\chi_{u}^{k} \wedge \chi_{v}^{m-k} \wedge \alpha^{n-m} \geq f^{\frac{k}{m}} g^{\frac{m-k}{m}} \alpha^{n} .
$$

Proof. It is a simple consequence of the mixed type inequality in the smooth case, and then for continuous functions we use Theorem 1.1 and Lemma 3.13.

Thanks to this type of inequality with $\chi=\alpha=\omega$ we are able to relax the smoothness assumption on potentials in the statement of [43, Proposition 3.16]. In particular, the uniqueness of continuous solutions to the complex Hessian equation on compact Hermitian manifolds with strictly positive right hand side in $L^{p}, p>$ $n / m$.

Corollary 4.11. Let $(X, \omega)$ be a compact Hermitian manifold. Suppose that $u, v \in$ $S H_{m}(\omega) \cap C^{0}(X), \sup _{X} u=\sup _{X} v=0$, satisfy

$$
\omega_{u}^{m} \wedge \omega^{n-m}=f \omega^{n}, \quad \omega_{v}^{m} \wedge \omega^{n-m}=g \omega^{n},
$$

where $f, g \in L^{p}\left(X, \omega^{n}\right), p>n / m$. Assume that

$$
f \geq c_{0}>0
$$

for some constant $c_{0}$. Fix $0<a<\frac{1}{m+1}$. Then,

$$
\|u-v\|_{L^{\infty}} \leq C\|f-g\|_{L^{p}}^{a},
$$

where the constant $C$ depends on $c_{0}, a, p,\|f\|_{L^{p}},\|g\|_{L^{p}}, \omega, X$.

We can also show that continuous solutions obtained in [43] are also the continuous solutions in the viscosity sense and vice versa ( $\mathrm{Lu}$ [48 proved the existence and uniqueness of viscosity solutions to the complex Hessian equation on some special compact Hermitian manifolds). The viscosity approach for the Monge-Ampère equation on Kähler manifolds was used by Eyssidieux, Guedj, Zeriahi [22], Wang 68. It seems to be interesting to investigate the viscosity method for the complex Hessian equation on compact Hermitian manifolds with or without boundary. We refer the readers to [32, Example 18.1], 33, Example 3.2.7] for some results in this direction.

\section{Proof of Theorem 1.1}

In this section we proceed to prove Theorem 1.1, which we used in Sections 3 , 4. The proof is independent of results in those sections.

Let us rewrite the equation in the PDE form as in the paper by Székelyhidi 62 . Without loss of generality we fix $\Omega:=B(0, \delta) \subset B(0,1) \subset \mathbb{C}^{n}$ for $0<\delta<<1$. Let $\alpha$ be a Hermitian metric in $B(0,1)$. Fix a smooth real $(1,1)$-form $\chi$ on $B(0,1)$. For a $C^{2}$ function $u$ we consider the real $(1,1)$-form $g=\chi+\sqrt{-1} \partial \bar{\partial} u$, i.e., $g_{i \bar{j}}=\chi_{i \bar{j}}+u_{i \bar{j}}$. We can define $A_{j}^{i}:=\alpha^{\bar{p} i} g_{j \bar{p}}$, where $\alpha^{\bar{j} i}$ is the inverse of $\alpha_{i \bar{j}}$. Then, the matrix $A_{j}^{i}$ is 
Hermitian with respect to the metric $\alpha$, i.e., $A \times\left[\alpha_{i \bar{j}}\right]$ is a Hermitian matrix. Denote $\lambda(A)=\left(\lambda_{1}, \ldots, \lambda_{n}\right) \in \mathbb{R}^{n}$ the $n$-tuple of eigenvalues of $A$. In other words, $\lambda$ is the eigenvector of $g_{i \bar{j}}$ with respect to the metric $\alpha$. The complex Hessian equation (1.1) is

$$
F(A)=h,
$$

where

$$
F(A):=f(\lambda(A))=\left[\left(S_{m}(\lambda)\right]^{1 / m},\right.
$$

and $f$ is a symmetric increasing concave function defined on the cone $\Gamma_{m}$. Recall that the $m$-th elementary symmetric cone is

$$
\Gamma_{m}=\left\{\lambda \in \mathbb{R}^{n}: S_{1}(\lambda)>0, \ldots, S_{m}(\lambda)>0\right\} .
$$

Fix $0<h \in C^{\infty}(\bar{\Omega})$ and a smooth boundary data $\varphi \in C^{\infty}(\partial \Omega)$. We wish to study the Dirichlet problem, seeking $u \in C^{\infty}(\bar{\Omega})$ and $u=\varphi$ smooth on $\partial \Omega$ such that

$$
\left\{\begin{array}{l}
\lambda(A) \quad \in \Gamma_{m}, \\
F(A)=h
\end{array}\right.
$$

where $A_{j}^{i}=\alpha^{\bar{p} i}\left(\chi_{j \bar{p}}+u_{j \bar{p}}\right)$. To simplify notation, first we extend $\varphi \in C^{\infty}(\partial \Omega)$ smoothly to $\overline{B(0,1)}$. Upon replacing

$$
\begin{array}{r}
\tilde{u}:=u-C\left(|z|^{2}-\delta^{2}\right)-\varphi, \\
\tilde{\chi}:=\chi+\sqrt{-1} \partial \bar{\partial}\left[C\left(|z|^{2}-\delta^{2}\right)+\varphi\right],
\end{array}
$$

with $C>0$ large enough, which does not change $g_{i \bar{j}}$, we may assume that

$$
u=0 \quad \text { on } \partial \Omega, \quad \chi \geq \alpha \quad \text { on } \bar{\Omega}
$$

and 0 is the subsolution, i.e., $\chi^{m} \wedge \alpha^{n-m} \geq h$.

Let $F^{i j}(A):=\partial F / \partial a_{i j}$ be the partial derivative of $F$ at $A$ with respect to entry $a_{i j}$. We also denote

$$
\mathcal{F}:=\sum_{1 \leq i \leq n} f_{i},
$$

where $f_{i}=\partial f / \partial \lambda_{i}>0$ are precisely eigenvalues of $F^{i j}$ with respect to metric $\alpha$. If we choose coordinates in which $\alpha$ is orthonormal and $A$ being diagonal, then

$$
F^{i j}=\delta_{i j} f_{i},
$$

and thus $\mathcal{F}=\sum_{i=1}^{n} F^{i i}$.

We will proceed in Sections 6, 7, 8 to get a priori estimates, up to second order, and using the results in Tosatti-Weinkove-Wang-Yang 64, to get $C^{2, \alpha}$ interior estimates. This combined with the $C^{2}$-estimates at the boundary thus gives the full $C^{2}$ estimates up to the boundary of the real Hessian of $u$. This allows us to apply Krylov's boundary estimate 44 to get the desired $C^{2, \alpha}(\bar{\Omega})$ estimate. The higher order estimates are obtained by the bootstrapping argument, and then using the continuity method to obtain a solution to the equation (5.1). The uniqueness follows from the maximum principle. 
6. $C^{0}$-estimate

Denote $B_{j}^{i}=\alpha^{\bar{p} i} \chi_{j \bar{p}}$. Then, $F(A)=h \leq F(B)$ and $u \geq 0$ on $\partial \Omega$. Solve the linear PDE

$$
\left\{\begin{array}{ll}
n\left(\chi+\sqrt{-1} \partial \bar{\partial} u_{1}\right) \wedge \alpha^{n-1} / \alpha^{n} & =0, \\
u_{1} & =0
\end{array} \text { on } \partial \Omega .\right.
$$

By the maximum principle we get that for some $C_{0}>0$,

$$
0 \leq u \leq u_{1} \leq C_{0} .
$$

As $u=u_{1}=0$ on $\partial \Omega$, it also follows that for some $C_{0}^{\prime}>0$,

$$
\begin{aligned}
& |\nabla u| \leq C_{0}^{\prime} \quad \text { on } \partial \Omega . \\
& \text { 7. } C^{1}-\text { ESTIMATE }
\end{aligned}
$$

In this section we prove the gradient estimate. Here the assumption of small radius is important. (Notice that Plis [59] has claimed this estimate in the case $\chi \equiv 0$ and $\alpha$ Kähler for any ball but no proof was given there.)

By (5.2) we may suppose that for some $C_{1}>0$,

$$
\begin{gathered}
\frac{\delta_{i j}}{C_{1}} \leq \alpha_{i \bar{j}} \leq \chi_{i \bar{j}} \leq C_{1} \delta_{i j} . \\
L:=\sup _{\Omega}|u|+1 .
\end{gathered}
$$

Let $\nabla$ denote the Chern connection with respect to $\alpha$. Note that $\|z\|_{\alpha}^{2}$ is strictly plurisubharmonic as long as $\delta$ small. More precisely, we choose $\delta$ so that

$$
\nabla_{\bar{p}} \nabla_{p}\|z\|_{\alpha}^{2}=\partial_{\bar{p}} \partial_{p}\left(\alpha_{i \bar{j}} z^{i} \bar{z}^{j}\right)=\alpha_{p \bar{p}}+O(|z|) \geq \alpha_{p \bar{p}} / 2 .
$$

Denote $v=N\left(\sup _{z \in \Omega}\|z\|_{\alpha}^{2}-\|z\|_{\alpha}^{2}\right)$, where $N>0$ is a constant to be determined later. We see that

$$
0 \leq v \leq N C_{1} \delta^{2} \quad \text { and } \quad-v_{p \bar{p}}=-\partial_{p} \partial_{\bar{p}} v \geq N / 2 C_{1} .
$$

Consider

with

$$
G=\log \|\nabla u\|_{\alpha}^{2}+\psi(u+v)
$$

$$
\psi(t)=-\frac{1}{2} \log \left(1+\frac{t}{L+N C_{1} \delta^{2}}\right) .
$$

Note that a similar function was considered by Hou-Ma-Wu [36] and it satisfies

$$
\psi^{\prime}<0, \quad \psi^{\prime \prime}=2 \psi^{\prime 2} .
$$

If $G$ attains its maximum at a boundary point, then $\sup _{\Omega}|\nabla u|$ is uniformly bounded by $\sup _{\partial \Omega}|\nabla u|$, up to a uniform constant. By (6.2), the latter one is uniformly bounded. Then, we will get the $C^{1}$ - estimate. Therefore, we may assume that the maximum point belongs to $\Omega$. We shall derive the desired estimate by using maximum principle at this point.

We choose the orthonormal coordinates for $\alpha$ such that at this point $\alpha_{i \bar{j}}$ is the identity matrix and $A_{j}^{i}$ is diagonal. All computations bellow are performed at this point and the subscripts stand for usual derivatives if there is no otherwise indication. 
Differentiating $G$ twice and evaluating the equations at the maximum point we have:

$$
\begin{gathered}
G_{p}=\frac{\left(\nabla_{p} \nabla_{i} u\right) u_{\bar{i}}+u_{i} \nabla_{p} \nabla_{\bar{i}} u}{|\nabla u|^{2}}+\psi^{\prime}\left(u_{p}+v_{p}\right)=0 ; \\
G_{p \bar{p}}=\frac{\left(\nabla_{\bar{p}} \nabla_{p} \nabla_{i} u\right) u_{\bar{i}}+u_{i} \nabla_{\bar{p}} \nabla_{p} \nabla_{\bar{i}} u+\left|\nabla_{p} \nabla_{i} u\right|^{2}+\left|\nabla_{\bar{p}} \nabla_{i} u\right|^{2}}{|\nabla u|^{2}} \\
-\frac{1}{|\nabla u|^{4}}\left|u_{i} \nabla_{p} \nabla_{\bar{i}} u+u_{\bar{i}} \nabla_{p} \nabla_{i} u\right|^{2} \\
+\psi^{\prime \prime}\left|u_{p}+v_{p}\right|^{2}+\psi^{\prime}\left(u_{p \bar{p}}+v_{p \bar{p}}\right) .
\end{gathered}
$$

Next, we have

$$
\begin{aligned}
\nabla_{\bar{p}} \nabla_{p} \nabla_{i} u & =u_{p \bar{p} i}-\left(\partial_{\bar{p}} \Gamma_{p i}^{q}\right) u_{q}-\Gamma_{p i}^{q} u_{q \bar{p}} \\
& =g_{p \bar{p} i}-\chi_{p \bar{p} i}-\left(\partial_{\bar{p}} \Gamma_{p i}^{q}\right) u_{q}-\Gamma_{p i}^{p} \lambda_{p}+\Gamma_{p i}^{q} \chi_{q \bar{p}},
\end{aligned}
$$

where we used that $g_{i j}$ is diagonal. Similarly,

$$
\begin{aligned}
\nabla_{\bar{p}} \nabla_{p} \nabla_{\bar{i}} u & =u_{p \bar{p} \bar{i}}-\overline{\Gamma_{p i}^{q}} u_{p \bar{q}} \\
& =g_{p \bar{p} \bar{i}}-\chi_{p \bar{p} \bar{i}}-\overline{\Gamma_{p i}^{p}} \lambda_{p}+\overline{\Gamma_{p i}^{q}} \chi_{p \bar{q}} .
\end{aligned}
$$

Moreover, by applying the covariant derivatives to the equation we get

$$
F^{p p} \nabla_{i} g_{p \bar{p}}=h_{i} .
$$

As $\nabla_{i} g_{p \bar{p}}=g_{p \bar{p} i}-\Gamma_{i p}^{m} g_{m \bar{p}}$, we have $F^{p p} g_{p \bar{p} i}=h_{i}+F^{p p} \Gamma_{i p}^{p} \lambda_{p}$. Combining with (17.7) we get that

$$
\begin{aligned}
F^{p p}\left(\nabla_{\bar{p}} \nabla_{p} \nabla_{i} u\right) u_{\bar{i}}= & h_{i} u_{\bar{i}}+F^{p p}\left(\Gamma_{i p}^{p}-\Gamma_{p i}^{p}\right) \lambda_{p} u_{\bar{i}}-F^{p p} \chi_{p \bar{p} i} u_{\bar{i}} \\
& -F^{p p}\left(\partial_{\bar{p}} \Gamma_{p i}^{q}\right) u_{q} u_{\bar{i}}+F^{p p} \Gamma_{p i}^{q} \chi_{q \bar{p}} u_{\bar{i}} .
\end{aligned}
$$

Similarly,

$$
\begin{aligned}
F^{p p}\left(\nabla_{\bar{p}} \nabla_{p} \nabla_{\bar{i}} u\right) u_{i}= & h_{\bar{i}} u_{i}+F^{p p}\left(\overline{\Gamma_{i p}^{p}}-\overline{\Gamma_{p i}^{p}}\right) \lambda_{p} u_{i} \\
& -F^{p p} \chi_{p \bar{p} \bar{\imath}} u_{i}-F^{p p} \overline{\Gamma_{p i}^{q}} \chi_{p \bar{q}} u_{i}
\end{aligned}
$$

Let's denote

$$
R:=\sup _{p, q, i}\left|\partial_{\bar{p}} \Gamma_{p i}^{q}\right|, \quad T:=\sup _{i, p}\left|\Gamma_{i p}^{p}-\Gamma_{p i}^{p}\right|,
$$

which are bounds for the curvature and torsion of metric $\alpha$ on $\bar{B}(0,1)$.

It follows from (7.8) and (7.9) that, for $K:=|\nabla u|^{2}$ large enough,

$$
\begin{aligned}
& \frac{1}{K} F^{p p}\left[\left(\nabla_{\bar{p}} \nabla_{p} \nabla_{i} u\right) u_{\bar{i}}+\left(\nabla_{\bar{p}} \nabla_{p} \nabla_{\bar{i}} u\right) u_{i}\right] \\
& \geq-C / K^{1 / 2}-F^{p p}\left|\lambda_{p}\right| T / K^{1 / 2}-C \mathcal{F} / K^{1 / 2}-R \mathcal{F} \\
& \geq-C-\frac{1}{2 K} F^{p p} \lambda_{p}^{2}-\left(R+T^{2}+1\right) \mathcal{F},
\end{aligned}
$$

where in the last inequality we used

$$
\frac{\left|\lambda_{p}\right| T}{K^{1 / 2}} \leq \frac{1}{2}\left(\frac{\lambda_{p}^{2}}{K}+T^{2}\right)
$$

By the equation (7.5)

$$
-\frac{1}{K^{2}}\left|u_{i} \nabla_{p} \nabla_{\bar{i}} u+u_{\bar{i}} \nabla_{p} \nabla_{i} u\right|^{2}=-\psi^{\prime 2}\left|u_{p}+v_{p}\right|^{2} .
$$


By $\sum_{p=1}^{n} f_{p} \lambda_{p}=h$ and $\chi_{p \bar{p}} \geq 1$ we have

$$
\begin{aligned}
\psi^{\prime} F^{p p}\left(u_{p \bar{p}}+v_{p \bar{p}}\right) & =\psi^{\prime} F^{p p} \lambda_{p}+\left|\psi^{\prime}\right| F^{p p}\left[\chi_{p \bar{p}}+\left(-v_{p \bar{p}}\right)\right] \\
& \geq-C+\left|\psi^{\prime}\right|\left[1+N / 2 C_{1}\right] \mathcal{F}
\end{aligned}
$$

We also note that

$$
\begin{aligned}
\frac{1}{K} F^{p p}\left|\nabla_{\bar{p}} \nabla_{i} u\right|^{2} & =\frac{1}{K} F^{p p}\left|g_{i \bar{p}}-\chi_{i \bar{p}}\right|^{2} \\
& \geq \frac{1}{2 K} F^{p p}\left|\lambda_{p}\right|^{2}-\frac{1}{K} F^{p p}\left|\chi_{i \bar{p}}\right|^{2} \\
& \geq \frac{1}{2 K} F^{p p}\left|\lambda_{p}\right|^{2}-\frac{C \mathcal{F}}{K} .
\end{aligned}
$$

Therefore, combining (7.6), (7.10), (7.11), (7.12) and (7.13), we get that

$$
\begin{aligned}
0 \geq F^{p p} G_{p \bar{p}} \geq & -C-\frac{1}{2 K} F^{p p}\left|\lambda_{p}\right|^{2}-\left(R+T^{2}+1\right) \mathcal{F} \\
& +\frac{1}{2 K} F^{p p}\left|\lambda_{p}\right|^{2}-\frac{C \mathcal{F}}{K} \\
& +\left(\psi^{\prime \prime}-\psi^{\prime 2}\right) F^{p p}\left|u_{p}+v_{p}\right|^{2} \\
& +\left|\psi^{\prime}\right|\left[1+N / 2 C_{1}\right] \mathcal{F} .
\end{aligned}
$$

We may assume that $K>C$. As $\psi^{\prime \prime}=2 \psi^{\prime 2}$, we simplify the inequality:

$$
0 \geq \psi^{\prime 2} F^{p p}\left|u_{p}+v_{p}\right|^{2}+\left|\psi^{\prime}\right|\left(1+N / 2 C_{1}\right) \mathcal{F}-\left(R+T^{2}+2\right) \mathcal{F}-C .
$$

Now we decrease further $\delta$ (if necessary) so that $16\left(R+T^{2}+3\right) C_{1}^{2} \delta^{2}<1$. Hence, we can choose $N>1$ satisfying

$$
\frac{N}{8\left(L C_{1}+N C_{1}^{2} \delta^{2}\right)} \geq R+T^{2}+3
$$

On the interval $t \in\left[0, L+N C_{1} \delta^{2}\right]$, we have $\left|\psi^{\prime}\right| \geq 1 / 4\left(L+N C_{1} \delta^{2}\right)$. Hence,

$$
\frac{N\left|\psi^{\prime}\right|}{2 C_{1}} \geq R+T^{2}+3
$$

It follows from (7.14) and (7.15) that

$$
F^{p p}\left|u_{p}+v_{p}\right|^{2}+\mathcal{F} \leq C,
$$

where $C=C\left(A, C_{1}, L\right)$. We shall use (7.16) to prove that

$$
F^{i i}=\frac{S_{m}^{-1+1 / m}(\lambda)}{m} S_{m-1 ; i}(\lambda) \geq c>0
$$

for some uniform $c$ and for every $1 \leq i \leq n$. Indeed, since

$$
\mathcal{F}=\frac{S_{m}^{-1+1 / m}(\lambda)}{m} \sum_{i=1}^{n} S_{m-1 ; i}(\lambda) \leq C,
$$

we have $S_{m-1 ; i}(\lambda) \leq C$ for every $i=1, \ldots, n$. By the inequality [67, Proposition 2.1 (4)]

$$
\prod_{i=1}^{n} S_{m-1 ; i}(\lambda) \geq C_{n, m}\left[S_{m}(\lambda)\right]^{n(m-1) / m}
$$


where $C_{n, m}>0$ depends only on $n, m$. Thus, the desired lower bound for each $S_{m-1 ; i}(\lambda)$ follows from the equation $\left(S_{m}(\lambda)\right)^{\frac{1}{m}}=h>0$ and the upper bound for $S_{m-1 ; i}(\lambda)$. We also get the lower bound for each $F^{i i}$. Finally, from

$$
F^{p p}\left|u_{p}+v_{p}\right|^{2} \leq C
$$

we easily get the a priori gradient bound, $|\nabla u| \leq C$.

$$
\text { 8. } C^{2} \text { - Estimates }
$$

In this section we prove the following estimate

$$
\sup _{\bar{\Omega}}|\sqrt{-1} \partial \bar{\partial} u| \leq C
$$

where $C$ depends on $\|u\|_{L^{\infty}(\bar{\Omega})},\|\nabla u\|_{L^{\infty}(\bar{\Omega})}$ and the given data.

If $\sup _{\bar{\Omega}}|\partial \bar{\partial} u|$ is attained at an interior point of $\Omega$, then by a result of Székelyhidi 62. (see also Zhang [69]) we have for some $C>0$, which depends on $\|u\|_{\infty}$ and the given data,

$$
|\sqrt{-1} \partial \bar{\partial} u| \leq C\left(1+\sup _{\bar{\Omega}}|\nabla u|^{2}\right) .
$$

Therefore, we only need to consider the case when the maximum point $P$ is on the boundary. At this point, following Boucksom [8], we choose a local half-ball coordinate $U$ such that $z(P)=0$ and $r$ is the defining function for $U \cap \partial \Omega$. Then, $U \cap \Omega=\{r \leq 0\} \cap \Omega$. We choose the coordinates $z=\left(z_{1}, \ldots, z_{n}\right)$, centred at 0 , such that the positive $x_{n}$ axis is the interior normal direction, and near 0 the graph $U \cap \partial \Omega$ is written as

$$
r=-x_{n}+\sum_{j, k=1}^{n} a_{j k} z_{j} \bar{z}_{k}+O\left(|z|^{3}\right)=0
$$

We refer the reader to the expository paper of Boucksom [8] for more details on this coordinate.

Recall that $\lambda_{i}$ 's are eigenvalue functions of matrix $A$, i.e.

$$
\lambda(A)=\left(\lambda_{1}, \ldots, \lambda_{n}\right) .
$$

We often represent quantities in the orthonormal coordinates $\left(w^{1}, . ., w^{n}\right)$ in which $\alpha_{i \bar{j}}$ is the identity and $A_{j}^{i}$ is diagonal. The following equations will help us in computing quantities in the orthonormal coordinates once we know theirs forms in the fixed coordinates $\left(z^{1}, \ldots, z^{n}\right)$.

Suppose at a given point we change the coordinates, $w=X z$, i.e.

$$
w^{i}=x_{i k} z^{k}, \quad x_{i k} \in \mathbb{C},
$$

and we obtain at that point

$$
\begin{aligned}
& \alpha_{i \bar{j}} \sqrt{-1} d z^{i} \wedge d \bar{z}^{j}=\sum_{a=1}^{n} \sqrt{-1} d w^{a} \wedge d \bar{w}^{a} \\
& g_{i \bar{j}} \sqrt{-1} d z^{i} \wedge d \bar{z}^{j}=\sum_{a=1}^{n} \lambda_{a} \sqrt{-1} d w^{a} \wedge d \bar{w}^{a} .
\end{aligned}
$$

It follows that

$$
\alpha_{i \bar{j}}=x_{a i} \overline{x_{a j}}, \quad g_{i \bar{j}}=x_{a i} \lambda_{a} \overline{x_{a j}} .
$$


It is clear that for every $1 \leq i \leq n$,

$$
\sum_{a=1}^{n}\left|x_{a i}\right|^{2}=\alpha_{i \bar{i}}<C .
$$

Moreover, the inverse of matrix $\alpha_{i \bar{j}}$ is given by the formula

$$
\alpha^{\overline{j i}}=\bar{x}^{j a} x^{i a}
$$

where $x^{i a}$ is the inverse of $X$. Hence,

$$
A_{j}^{i}=\alpha^{\bar{p} i} g_{j \bar{p}}=x^{i a} \lambda_{a} x_{a j}
$$

In $\mathbb{C}^{n \times n}$ if we change coordinates $B=X A X^{-1}=\left(b_{k l}\right)$, then at the considered point $B$ is a diagonal matrix $\left(\lambda_{1}, \ldots, \lambda_{n}\right)$. Therefore, $\lambda_{a}$ is smooth at the diagonal matrix $B$ (see e.g. [61]) and

$$
\begin{gathered}
\frac{\partial F}{\partial b_{k l}}=\frac{\partial f}{\partial \lambda_{a}} \cdot \frac{\partial \lambda_{a}}{\partial b_{k l}}=f_{a} \delta_{a k} \delta_{a l} \\
\frac{\partial F}{\partial a_{i j}}=\frac{\partial F}{\partial b_{k l}} \frac{\partial b_{k l}}{\partial a_{i j}}=\sum_{k, l} \sum_{a=1}^{n} f_{a} \delta_{a k} \delta_{a l} x_{k i} x^{j l}=x^{j a} f_{a} x_{a i} .
\end{gathered}
$$

An easy consequence from the above formula is that

$$
L^{\bar{p} j}:=F^{i j} \alpha^{\bar{p} i}=\overline{x^{p a}} f_{a} x^{j a},
$$

where $F^{i j}=\partial F / \partial a_{i j}$ at $A_{j}^{i}$, is a positive definite Hermitian matrix.

To derive the desired a priori estimate we will use the linearised elliptic operator, for a smooth function $w$,

$$
L w:=L^{\bar{p} j} \partial_{j} \partial_{\bar{p}} w=F^{i j} \alpha^{\bar{p} i} \partial_{j} \partial_{\bar{p}} w,
$$

It is worth to recall that

$$
\mathcal{F}:=\sum_{1 \leq i \leq n} f_{i}
$$

where $f_{i}=\partial f / \partial \lambda_{i}$ are eigenvalues of $F^{i j}$ with respect to metric $\alpha$.

Following Guan [26] (c.f. Boucksom 8]) we construct the important barrier function.

Lemma 8.1. Set $b=u-r-\mu r^{2}$. Then, there exist constants $\mu>0$ and $\tau>0$ such that

$$
L b \leq-\frac{1}{2} \mathcal{F}
$$

and $b \geq 0$ on the half-ball coordinate $U$ of radius $|r|<\tau$.

Proof. By shrinking the radius of the half coordinate ball $U$, we have $r$ is plurisubharmonic in $U$. Then

$$
0 \leq L r=L^{\bar{p} j} r_{j \bar{p}} \leq C \mathcal{F}
$$

As $b_{j \bar{p}}:=\partial_{j} \partial_{\bar{p}} b$ is a Hermitian matrix and $\alpha_{i \bar{j}}>0$, we can represent

$$
b_{j \bar{p}}=x_{a j} \gamma_{a} \overline{x_{a p}}
$$

where $\gamma_{a} \in \mathbb{R}$ are eigenvalues of $b_{i \bar{j}}$ with respect to the matrix $\alpha_{i \bar{j}}$. Hence,

$$
L b=\sum_{a=1}^{n} f_{a} \gamma_{a}
$$


which does not depend on the choice of coordinates of $\alpha$. Thus, to verify the desired inequality at a given point, we compute, at this point, in orthonormal coordinates of $\alpha$ and $A_{j}^{i}=\alpha^{\bar{p} i}\left(\chi_{j \bar{p}}+u_{j \bar{p}}\right)=\left(\lambda_{1}, \ldots, \lambda_{n}\right)$ diagonal. So is $L^{\bar{p} i}=\left(f_{1}, \ldots, f_{n}\right)$.

We now compute, as $r \leq 0$,

$$
\begin{aligned}
L b & =L^{\bar{i} i} u_{i \bar{i}}-L r-2 \mu r L r-2 \mu L^{\bar{i} i}\left|r_{i}\right|^{2} \\
& =L^{\bar{i} i} g_{i \bar{i}}-L^{\bar{i} i} \chi_{i \bar{i}}-L r+2 \mu|r| L r-2 \mu L^{\bar{i} i} r_{i}^{2} \\
& =\sum_{i=1}^{n} f_{i} \lambda_{i}+(2 \mu|r|-1) L r-L^{\bar{i} i}\left(\chi_{i \bar{i}}+2 \mu r_{i}^{2}\right) .
\end{aligned}
$$

We have $\sum_{i=1}^{n} f_{i} \lambda_{i}=h$ and

$$
(2 \mu|r|-1) L r \leq 2 C \mu|r| \mathcal{F} \text {. }
$$

Notice that $\chi_{i \bar{i}} \geq \alpha_{i \bar{i}}=1$. The last negative term (8.4) will be divided into three parts. First

$$
-L^{\bar{i} i} \chi_{i \bar{i}} / 2 \leq-\mathcal{F} / 2 \text {. }
$$

Next, we use $-L^{\bar{i} i} \chi_{i \bar{i}} / 4$ to absorb the right hand side of (8.5) (i.e. the second term in (8.4) ), provided that

$$
C \mu|r| \leq 1 / 8
$$

We will use the part $-L^{\bar{i} i}\left(\frac{\chi_{i \bar{i}}}{4}+2 \mu r_{i}^{2}\right)$ for $\mu$ large to absorb the first term in (8.4). We claim that

$$
L^{\bar{i} i}\left(\frac{\chi_{i \bar{i}}}{4}+2 \mu r_{i}^{2}\right) \geq c_{0} \mu^{\frac{1}{m}}
$$

for some uniform $c_{0}>0$. In fact, if $m=1$, then it is obvious. We may assume that $m>1$. Observe that $|\nabla r|>0$ at 0 , then decrease $\tau$ if necessary, we have

$$
|\nabla r|^{2}=\sum_{i=1}^{n} r_{i}^{2}>c_{1}
$$

for a uniform $c_{1}>0$ on $U$. By Gårding's inequality [25, Theorem 5] with $\lambda^{\prime}=$ $\left(\frac{\chi_{1 \overline{1}}}{4}+2 \mu r_{1}^{2}, \ldots, \frac{\chi_{n \bar{n}}}{4}+2 \mu r_{n}^{2}\right)$ and $S_{m-1 ; i}(\lambda)$, we have

$$
\begin{aligned}
\sum_{i=1}^{n}\left(\frac{\chi_{i \bar{i}}}{4}+2 \mu\left|r_{i}\right|^{2}\right) S_{m-1 ; i}(\lambda) & \geq m\left[S_{m}\left(\chi_{i \bar{i}} / 4+2 \mu\left|r_{i}\right|^{2}\right)\right]^{\frac{1}{m}}\left[S_{m}(\lambda)\right]^{\frac{m-1}{m}} \\
& \geq \frac{m \mu^{\frac{1}{m}} h^{m-1}}{4^{\frac{m-1}{m}}}\left(\sum_{i=1}^{n} 2\left|r_{i}\right|^{2} \prod_{k \neq i} \chi_{k \bar{k}}\right)^{\frac{1}{m}} \\
& \geq \frac{m \mu^{\frac{1}{m}} h^{m-1}}{4^{\frac{m-1}{m}}}\left(\sum_{i=1}^{n} 2\left|r_{i}\right|^{2}\right)^{\frac{1}{m}} \\
& \geq \frac{2^{\frac{1}{m}} m \mu^{\frac{1}{m}} h^{m-1}}{4^{\frac{m-1}{m}}} c_{1}^{\frac{1}{m}}
\end{aligned}
$$

where we used $\chi_{k \bar{k}} \geq 1$ for the third inequality and used (8.7) for the last inequality.

To obtain the inequality (8.6), we only need to notice that

$$
L^{\bar{i} i}=f_{i}=\frac{\left[S_{m}(\lambda)\right]^{(1-m) / m} S_{m-1 ; i}(\lambda)}{m} .
$$


Therefore, the uniform constant we get is $c_{0}=C\left(c_{1}, h, m\right)>0$. So we can choose $\mu>0$ large enough to get the desired inequality for $L b$.

It remains to check that $b \geq 0$. Since $u \geq 0$ it is enough to have that

$$
-r-\mu r^{2}=|r|(1-\mu|r|) \geq 0 .
$$

This easily follows by further decreasing (if necessary) the radius $\tau$ of the half-ball coordinate.

We are ready to prove the second order estimates for $u$ at the boundary point $0 \in \partial \Omega$. Following Caffarelli, Nirenberg, Kohn, Spruck [12] (c.f [8]) we set

$$
t_{1}=x_{1}, t_{2}=y_{1}, \ldots, t_{2 n-2}=y_{n-1}, t_{2 n-1}=y_{n}, t_{2 n}=x_{n} .
$$

Let $D_{1}, \ldots, D_{2 n}$ be the dual basis of $d t_{1}, \ldots, d t_{2 n-1},-d r$, then

and

$$
D_{j}=\frac{\partial}{\partial t_{j}}-\frac{r_{t_{j}}}{r_{x_{n}}} \frac{\partial}{\partial x_{n}} \quad \text { for } \quad 1 \leq j<2 n,
$$

$$
D_{2 n}=-\frac{1}{r_{x_{n}}} \frac{\partial}{\partial x_{n}}
$$

Because $u=0$ on $\partial \Omega$, we can write, for some positive function $\sigma$,

$$
u=\sigma r .
$$

Then,

$$
\partial u / \partial x_{n}(0)=-\sigma(0) .
$$

So, $|\sigma(0)|<C$. Moreover, for $1 \leq j \leq 2 n-1$,

$$
\frac{\partial^{2} u}{\partial t_{i} \partial t_{j}}(0)=\sigma(0) \frac{\partial^{2} r}{\partial t_{i} \partial t_{j}}(0)
$$

and hence tangential-tangential derivatives $\left|\partial_{t_{i}} \partial_{t_{j}} u\right|$ are under control.

Next, we bound normal-tangential derivatives:

Theorem 8.2. We have

$$
\left|\frac{\partial^{2} u}{\partial t_{j} \partial x_{n}}(0)\right| \leq C \quad \text { for } \quad j \leq 2 n-1
$$

where $C$ depends on $u,|\nabla u|$ and the given datum.

Proof. Without loss of generality we fix $j=1$ and we shall show that

$$
\left|D_{2 n} D_{1} u(0)\right| \leq C .
$$

The derivative $D_{1}$, acting on functions, is equal to

$$
\partial_{1}+\partial_{\overline{1}}+\tilde{r}\left(\partial_{n}+\partial_{\bar{n}}\right)
$$

where $\partial$ denotes the usual partial derivatives and $\tilde{r}:=-\frac{r_{x_{1}}}{r_{x_{n}}}$ is a smooth realvalued function near 0 . Recall that we use the subindex to denote usual derivatives in direction $\partial / \partial z_{1}, \ldots, \partial / \partial z_{n}$ and their conjugates if there is no other indication. This gives

$$
D_{1} u=u_{1}+u_{\overline{1}}+\tilde{r}\left(u_{n}+u_{\bar{n}}\right) .
$$

Following Caffarelli, Nirenberg, Spruck [13] and Guan [26, 27, our goal is to construct a function of form

$$
w=D_{1} u-\sum_{k<n}\left|u_{k}\right|^{2}-\left|u_{n}-u_{\bar{n}}\right|^{2}+\mu_{1} b+\mu_{2}|z|^{2},
$$


satisfying the following:

(i) $w(0)=0$;

(ii) $w \geq 0$ on $\partial U$;

(iii) $L w=L^{\bar{p} j} \partial_{j} \partial_{\bar{p}} w \leq 0$ in the interior of $U$,

where $b$ is the barrier function constructed in Lemma 8.1, constants $\mu_{1}, \mu_{2}>0$ are to be determined later.

To see the first property $(i)$ we note that, for $i<n$,

$$
2 u_{i}(0)=\frac{\partial u}{\partial x_{i}}(0)-\sqrt{-1} \frac{\partial u}{\partial y_{i}}(0)=0,
$$

and

$$
u_{n}(0)-u_{\bar{n}}(0)=-\sqrt{-1} \frac{\partial u}{\partial y_{n}}(0)=0 .
$$

Moreover, $D_{1} u(0)=b(0)=\tilde{r}(0)=0$. Therefore, the first property follows.

Next, we verify the second property $(i i)$. We claim that there exists a constant $\mu_{2}>0$ such that

$$
w \geq 0 \text { on } \partial U
$$

To see this consider two parts $\partial \Omega \cap U$ and $\partial U \backslash(\partial \Omega \cap U)$ of the boundary $\partial U$ separately.

Part 1: On $\partial \Omega \cap U$. We know that $D_{1} u=b=0$, and near 0

$$
x_{n}=\sum_{j, k=1}^{n} a_{j k} z_{j} \bar{z}_{k}+O\left(|z|^{3}\right) .
$$

By writing $x_{n}=\rho\left(t_{1}, \ldots, t_{2 n-1}\right)=\rho(t)$ we deduce that

$$
\rho(t)=\sum_{i, j<2 n} k_{i j} t_{i} t_{j}+O\left(|t|^{3}\right),
$$

where $\left(k_{i j}\right)=\left[\frac{\partial^{2} x_{n}}{\partial t_{i} \partial t_{j}}(0)\right]$ is uniformly bounded. Since $u(t, \rho(t))=0$,

$$
\partial u / \partial t_{i}+\partial u / \partial x_{n} \cdot \partial \rho / \partial t_{i}=0
$$

for $i<2 n$. Applying for $y_{n}=t_{2 n-1}$ gives

$$
\left|\partial u / \partial y_{n}\right|^{2} \leq C|t|^{2} \leq C|z|^{2} .
$$

Similarly, for $i<n$,

$$
\left|u_{i}\right|^{2} \leq C|z|^{2}
$$

Therefore, $w \geq 0$ on $\partial \Omega \cap U$ for $\mu_{2}>0$ large enough.

Part 2: On $\partial U \backslash(\partial \Omega \cap U)$. On this piece $|z|^{2}=\tau^{2}$ with $\tau$ being the radius of $U$. Since $b \geq 0$ on $U$, we have $w \geq 0$ as soon as

$$
\mu_{2} \tau^{2} \geq\left|D_{1} u\right|+\sum_{i=1}^{n}\left|u_{i}\right|^{2}
$$

This is done by choosing $\mu_{2}>0$ large as the right hand side is under control by the $C^{1}$-estimate. Thus, the second property is satisfied.

To verify the third property $(i i i), L^{\bar{p} j} w_{j \bar{p}} \leq 0$ in the interior of $U$, we fix an interior point $z_{0} \in U$. Below we compute at this fixed point. The estimation will be split into several steps. 
(1) Estimate for $D_{1} u$. We start by computing

$$
\begin{aligned}
L^{\bar{p} j}\left(D_{1} u\right)_{j \bar{p}}= & L^{\bar{p} j}\left[u_{1}+u_{\overline{1}}+\tilde{r}\left(u_{n}+u_{\bar{n}}\right)\right]_{j \bar{p}} \\
= & L^{\bar{p} j}\left[u_{1 j \bar{p}}+u_{\overline{1} j \bar{p}}+\tilde{r}\left(u_{n i \bar{p}}+u_{\bar{n} j \bar{p}}\right)\right] \\
& +L^{\bar{p} j}\left[\tilde{r}_{j}\left(u_{n}+u_{\bar{n}}\right)_{\bar{p}}+\tilde{r}_{\bar{p}}\left(u_{n}+u_{\bar{n}}\right)_{j}\right] \\
& +L^{\bar{p} j} \tilde{r}_{j \bar{p}}\left(u_{n}+u_{\bar{n}}\right) \\
= & : I_{1}+I_{2}+I_{3} .
\end{aligned}
$$

Let us denote $K:=\sup _{\Omega}|\nabla u|^{2}$, which is bounded by the $C^{1}$-estimate.

Lemma 8.3. There exists a constant $C$ depending only on $\alpha$ such that for any fixed $j, q$,

$$
\left|L^{\bar{p} j} g_{q \bar{p}}\right| \leq C \sum_{i=1}^{n} f_{i}\left|\lambda_{i}\right|
$$

Similarly,

$$
\left|L^{\bar{j} p} g_{p \bar{q}}\right| \leq C \sum_{i=1}^{n} f_{i}\left|\lambda_{i}\right|
$$

Proof. Recall that we have $\alpha_{i \bar{j}}=x_{a i} \overline{x_{a j}}, g_{i \bar{j}}=x_{a i} \lambda_{a} \overline{x_{a j}}$, and $L^{\bar{p} j}=\overline{x^{p a}} f_{a} x^{j a}$. Therefore,

$$
L^{\bar{p} j} g_{q \bar{p}}=\overline{x^{p a}} f_{a} x^{j a} x_{b q} \lambda_{b} \overline{x_{b p}}=x^{j a} f_{a} \lambda_{a} x_{a q} .
$$

Thus, the conclusion follows. The second inequality is proved in the same way.

(1a) Estimate $I_{2}$ and $I_{3}$. We first easily have

$$
\begin{aligned}
\left|I_{3}\right|=\left|L^{\bar{p} j} \tilde{r}_{j \bar{p}}\left(u_{n}+u_{\bar{n}}\right)\right| & \leq C K^{\frac{1}{2}} \mathcal{F} \\
& \leq C \mathcal{F} .
\end{aligned}
$$

Since two terms in $I_{2}$ are conjugate, so we will estimate one of them. We proceed as follows:

$$
\begin{aligned}
\tilde{r}_{j}\left(u_{n}+u_{\bar{n}}\right)_{\bar{p}} & =\tilde{r}_{j}\left[2 u_{n}-\left(u_{n}-u_{\bar{n}}\right)\right]_{\bar{p}} \\
& =2 \tilde{r}_{j} u_{n \bar{p}}-\tilde{r}_{j}\left(u_{n}-u_{\bar{n}}\right)_{\bar{p}} \\
& =2 \tilde{r}_{j} g_{n \bar{p}}-2 \tilde{r}_{j} \chi_{n \bar{p}}-\tilde{r}_{j} V_{\bar{p}},
\end{aligned}
$$

where we wrote $V=u_{n}-u_{\bar{n}}$.

By Lemma 8.3, we have for $\mathcal{F}|\lambda|:=\sum_{i} f_{i}\left|\lambda_{i}\right|$,

$$
\left|2 L^{\bar{p} j} \tilde{r}_{j} g_{n \bar{p}}\right| \leq C\left|L^{\bar{p} j} g_{n \bar{p}}\right| \leq C \mathcal{F}|\lambda| \text {. }
$$

A straightforward estimate gives

$$
\left|2 L^{\bar{p} j} \tilde{r}_{j} \chi_{n \bar{p}}\right| \leq C \mathcal{F} .
$$

Cauchy-Schwarz's inequality implies that

$$
\begin{aligned}
\left|L^{\bar{p} j} \tilde{r}_{j} V_{\bar{p}}\right| & \leq \frac{1}{2} L^{\bar{p} j} \tilde{r}_{j} \tilde{r}_{\bar{p}}+\frac{1}{2} L^{\bar{p} j}(\bar{V})_{j} V_{\bar{p}} \\
& \leq C \mathcal{F}+\frac{1}{2} L^{\bar{p} j}(\bar{V})_{j} V_{\bar{p}} .
\end{aligned}
$$

Thus, the above estimates give

$$
\left|I_{2}\right| \leq C(\mathcal{F}+\mathcal{F}|\lambda|)+L^{\bar{p} j}(\bar{V})_{j} V_{\bar{p}} .
$$


(1b) Estimate $I_{1}$. We have

$$
u_{1 j \bar{p}}=u_{j \bar{p} 1}=g_{j \bar{p} 1}-\chi_{j \bar{p} 1} .
$$

Covariant differentiation in direction $\partial / \partial z_{1}$ of the equation $F(A)=h$ gives

$$
F^{i j} \alpha^{\bar{p} i} \nabla_{1} g_{j \bar{p}}=L^{\bar{p} j}\left[g_{j \bar{p} 1}-\Gamma_{1 j}^{q} g_{q \bar{p}}\right]=h_{1} .
$$

It follows that

$$
\begin{aligned}
\left|L^{\bar{p} j} u_{1 j \bar{p}}\right| & =\left|L^{\bar{p} j}\left(g_{j \bar{p} 1}-\chi_{j \bar{p} 1}\right)\right| \\
& =\left|h_{k}+L^{\bar{p} j} \Gamma_{1 j}^{q} g_{q \bar{p}}-L^{\bar{p} j} \chi_{j \bar{p} 1}\right| \\
& \leq C(1+\mathcal{F})+\left|L^{\bar{p} j} \Gamma_{1 j}^{q} g_{q \bar{p}}\right| \\
& \leq C(1+\mathcal{F}+\mathcal{F}|\lambda|),
\end{aligned}
$$

where we used Lemma 8.3 for the last inequality.

The remaining terms in $I_{1}$ are estimated similarly, when the index 1 is replaced by $\overline{1}, \bar{n}$ or $n$. Therefore,

$$
\left|I_{1}\right| \leq C(1+\mathcal{F}+\mathcal{F}|\lambda|) .
$$

Combining (8.10), (8.11) and (8.14) yields

$$
\left|L^{\bar{p} j}\left(D_{1} u\right)_{j \bar{p}}\right| \leq C(1+\mathcal{F}+\mathcal{F}|\lambda|)+L^{\bar{p} j}(\bar{V})_{j} V_{\bar{p}} .
$$

We continue to estimate the other terms in the formula for $w$.

(2) Estimate for $-\sum_{k<n}\left|u_{k}\right|^{2}$. By computing

$$
\left(u_{k} u_{\bar{k}}\right)_{j \bar{p}}=u_{k j \bar{p}} u_{\bar{k}}+u_{k} u_{\bar{k} j \bar{p}}+u_{k j} u_{\bar{k} \bar{p}}+u_{k \bar{p}} u_{\bar{k} j}
$$

Similarly to the estimation of $I_{1}$, we have

$$
\begin{aligned}
\sum_{k<n}\left|L^{\bar{p} j}\left(u_{k j \bar{p}} u_{\bar{k}}+u_{k} u_{\bar{k} j \bar{p}}\right)\right| & \leq C K^{\frac{1}{2}}(1+\mathcal{F}+\mathcal{F}|\lambda|) \\
& \leq C(1+\mathcal{F}+\mathcal{F}|\lambda|) .
\end{aligned}
$$

For the third term, with $k$ fixed, $L^{\bar{p} j} u_{k j} u_{\bar{k} \bar{p}} \geq 0$. The last term in (8.16) will give a good positive term. By using Lemma 8.3 .

$$
\begin{aligned}
L^{\bar{p} j} u_{k \bar{p}} u_{\bar{k} j} & =L^{\bar{p} j}\left(g_{k \bar{p}}-\chi_{k \bar{p}}\right)\left(g_{j \bar{k}}-\chi_{j \bar{k}}\right) \\
& \geq L^{\bar{p} j} g_{k \bar{p}} g_{j \bar{k}}-C(\mathcal{F}+\mathcal{F}|\lambda|) .
\end{aligned}
$$

The following result is similar to Guan's [27, Proposition 2.19] in the real case.

Lemma 8.4. There exists an index s such that

$$
\sum_{k<n} L^{\bar{p} j} g_{k \bar{p}} g_{j \bar{k}} \geq \frac{\min _{i} \tau_{i}}{2} \sum_{i \neq s} f_{i} \lambda_{i}^{2}
$$

where $\tau_{i}$ 's are the eigenvalues of the matrix $\alpha_{i \bar{j}}$.

Proof of Lemma 8.4. First at the given point let $E=\left(e_{i j}\right)$ be a unitary matrix such that $\alpha=E^{t} \Lambda E$, where $\Lambda=\operatorname{diag}\left(\tau_{1}, \ldots, \tau_{n}\right)$. Without loss of generality, we can assume $X=\Lambda^{\frac{1}{2}} E$, so that $\alpha=X^{t} \bar{X}$ and $x_{i j}=\tau_{i}^{\frac{1}{2}} e_{i j}$. Again we have formulas $\alpha_{i \bar{j}}=x_{a i} \overline{x_{a j}}$ and $\alpha^{\bar{i} j}=\overline{x^{i a}} x^{j a}$. Moreover,

$$
L^{\bar{p} j}=\overline{x^{p a}} f_{a} x^{j a}, \quad g_{i \bar{j}}=x_{i b} \lambda_{b} \overline{x_{b j}} .
$$


Thus, for a fixed $k<n$,

$$
\begin{aligned}
L^{\bar{p} j} g_{k \bar{p}} g_{j \bar{k}} & =\overline{x^{p a}} f_{a} x^{j a} x_{b k} \lambda_{b} \overline{x_{b p}} x_{c j} \lambda_{c} \overline{x_{c k}} \\
& =\sum_{i=1}^{n} f_{i} \lambda_{i}^{2}\left|x_{i k}\right|^{2} .
\end{aligned}
$$

As

$$
\sum_{k<n}\left|x_{i k}\right|^{2}=\sum_{k=1}^{n}\left|x_{i k}\right|^{2}-\left|x_{i n}\right|^{2}=\tau_{i}\left(1-\left|e_{i n}\right|^{2}\right),
$$

we have

$$
S:=\sum_{k<n} L^{\bar{p} j} g_{k \bar{p}} g_{j \bar{k}}=\sum_{i=1}^{n} f_{i} \lambda_{i}^{2} \tau_{i}\left(1-\left|e_{i n}\right|^{2}\right) .
$$

If for every $1 \leq i \leq n$ we have $\left|e_{i n}\right|^{2} \leq \frac{1}{2}$, then

$$
S \geq \frac{\min _{i} \tau_{i}}{2} \sum_{i=1}^{n} f_{i} \lambda_{i}^{2}
$$

Otherwise, there exists an index $s$ such that $\left|e_{s n}\right|^{2}>\frac{1}{2}$. It follows that

$$
\sum_{i \neq s}\left|e_{i n}\right|^{2} \leq \frac{1}{2}
$$

Then,

$$
S=\sum_{i=1}^{n} f_{i} \lambda_{i}^{2} \tau_{i}\left(1-\left|e_{i n}\right|^{2}\right) \geq \sum_{i \neq s} f_{i} \lambda_{i}^{2} \tau_{i}\left(1-\left|e_{i n}\right|^{2}\right) \geq \frac{\min _{i} \tau_{i}}{2} \sum_{i \neq s} f_{i} \lambda_{i}^{2} .
$$

Thus, the lemma follows.

It follows from Lemma 8.4 and (8.17) that for some index $s$,

$$
\sum_{k<n} L^{\bar{p} j} u_{k \bar{p}} u_{\bar{k} j} \geq \frac{\min _{i} \tau_{i}}{2} \sum_{i \neq s} f_{i} \lambda_{i}^{2}-C(\mathcal{F}+\mathcal{F}|\lambda|) .
$$

Therefore,

$$
L\left(-\sum_{k<n}\left|u_{k}\right|^{2}\right) \leq-\frac{\min _{i} \tau_{i}}{2} \sum_{i \neq s} f_{i} \lambda_{i}^{2}+C(\mathcal{F}+\mathcal{F}|\lambda|) .
$$

(3) Estimate for $-|V|^{2}=-\left|u_{n}-u_{\bar{n}}\right|^{2}$. We compute

$$
\begin{aligned}
(V \bar{V})_{j \bar{p}}= & \left(u_{n j \bar{p}}-u_{\bar{n} j \bar{p}}\right) \bar{V}+V\left(u_{\bar{n} j \bar{p}}-u_{n j \bar{p}}\right) \\
& +V_{j}(\bar{V})_{\bar{p}}+(\bar{V})_{j} V_{\bar{p}} .
\end{aligned}
$$

Since $L^{\bar{p} j} V_{j}(\bar{V})_{\bar{p}} \geq 0$, we get, similarly to (8.13), the following

$$
L^{\bar{p} j}\left(-|V|^{2}\right)_{j \bar{p}} \leq-L^{\bar{p} j}(\bar{V})_{j} V_{\bar{p}}+C(1+\mathcal{F}+\mathcal{F}|\lambda|) .
$$

Combining (8.15), (8.18) and (8.19) gives us

$$
L w \leq-\frac{\min _{i} \tau_{i}}{2} \sum_{i \neq s} f_{i} \lambda_{i}^{2}+C(1+\mathcal{F}+\mathcal{F}|\lambda|)+\mu_{1} L b+\mu_{2} L\left(|z|^{2}\right) .
$$


By this and Lemma 8.1 we get that, for some index $s$,

$$
L w \leq-\frac{\min _{i} \tau_{i}}{2} \sum_{i \neq s} f_{i} \lambda_{i}^{2}+C \mathcal{F}|\lambda|+\left(C+\mu_{2}-\frac{\mu_{1}}{2}\right) \mathcal{F} .
$$

Recall that $\mu_{2}$ was chosen to have the property (ii) and $\mu_{1}>0$ can be chosen freely. To archive the third property of $w$ we need the following

Lemma 8.5. Let $\varepsilon>0$. There is a constant $C_{\varepsilon}>0$ such that for any index $s$,

$$
\mathcal{F}|\lambda|=\sum_{i=1}^{n} f_{i}\left|\lambda_{i}\right| \leq \varepsilon \sum_{i \neq s} f_{i} \lambda_{i}^{2}+C_{\varepsilon} \mathcal{F} .
$$

Proof of Lemma 8.5. Since $\sum_{i=1}^{n} f_{i} \lambda_{i}=h$, we have

$$
\begin{aligned}
\mathcal{F}|\lambda| & \leq 2 \sum_{i \neq s} f_{i}\left|\lambda_{i}\right|+h \\
& \leq \sum_{i \neq s} f_{i}\left(\varepsilon \lambda_{i}^{2}+\frac{1}{\varepsilon}\right)+h \\
& \leq \varepsilon \sum_{i \neq s} f_{i} \lambda_{i}^{2}+C_{\varepsilon} \mathcal{F},
\end{aligned}
$$

where we used the fact that $\mathcal{F}$ is uniformly bounded below by a positive constant.

Using Lemma 8.5 we get from (8.20) that

$$
L w \leq\left(-\frac{\min _{i} \tau_{i}}{2}+C \varepsilon\right) \sum_{i \neq s} f_{i} \lambda_{i}^{2}+\left(\mu_{2}+C+C_{\varepsilon}-\frac{\mu_{1}}{2}\right) \mathcal{F} .
$$

Thus, we choose $\varepsilon$ so small that the first term on the right hand side is negative, and then choose $\mu_{1}$ so large that the second term is also negative. The third property (iii) is proved.

We are ready to conclude the bound for tangential-normal second derivatives. By the maximum principle we have $w \geq 0$ on $U$. Therefore, as $w(0)=0, D_{2 n} w(0) \leq 0$. It follows that

$$
D_{2 n} D_{1} u(0) \leq C .
$$

The properties $(i),(i i)$ and (iii) also hold, with the same argument, if we replace $w$ by the function

$$
\tilde{w}=-D_{1} u-\sum_{k<n}\left|u_{k}\right|^{2}-\left|u_{n}-u_{\bar{n}}\right|^{2}+\mu_{1} b+\mu_{2}|z|^{2} .
$$

Therefore, $D_{2 n} D_{1} u(0) \geq-C$. Thus, we get the desired bound for $\left|D_{2 n} D_{1} u(0)\right|$.

The last estimate we need is the normal-normal derivative bound.

Lemma 8.6. We have

$$
\left|\frac{\partial^{2} u}{\partial x_{n}^{2}}(0)\right| \leq C,
$$

where $C$ depends on $h, C_{0}, C_{1}$, and the bounds of tangential-normal derivatives. 
Proof. Since $4 u_{n \bar{n}}=\partial^{2} u / \partial x_{n}^{2}+\partial^{2} u / \partial y_{n}^{2}$, the normal-normal estimate is equivalent to

$$
\left|u_{n \bar{n}}(0)\right| \leq C
$$

Moreover, as $\left|u_{i \bar{j}}\right|<C$ with $i+j<2 n$, we get that for $j<n$,

$$
\left|A_{j}^{i}\right|=\left|\alpha^{\bar{p} i}\left(\chi_{j \bar{p}}+u_{j \bar{p}}\right)\right|<C .
$$

Hence, it follows from

$$
\sum_{i=1}^{n} A_{i}^{i}=\sum_{i=1}^{n} \lambda_{i} \geq 0
$$

that $A_{n}^{n} \geq-C$, so is $g_{n \bar{n}} \geq-C$. It implies that $u_{n \bar{n}} \geq-C$. Therefore, it remains to prove that $u_{n \bar{n}} \leq C$. By $u=\sigma r$, with $\sigma>0$, we have for $j, k<n$,

$$
u_{j \bar{k}}(0)=\sigma(0) r_{j \bar{k}}(0) \text {. }
$$

Let $S$ be a $(n-1) \times(n-1)$ unitary matrix diagonalising $\left[u_{j \bar{k}}\right]_{j, k<n}$. It means that for $j, k<n$,

$$
u_{j \bar{k}}(0)=\sum_{p} S_{j p}^{*} d_{p} S_{p k}
$$

Since $r$ is strictly plurisubharmonic in $U$, we get that $d_{p}>0, p=1, \ldots, n-1$. By elementary matrix computation we have for $D=\left(d_{1}, . ., d_{n-1}\right)$ a diagonal matrix and the column vector $V=\left(u_{1 \bar{n}}, \ldots, u_{(n-1) \bar{n}}\right)^{t}$,

$$
\left(\begin{array}{cc}
S & 0 \\
0 & 1
\end{array}\right) \times\left[u_{i \bar{j}}\right]_{i, j \leq n} \times\left(\begin{array}{cc}
S^{*} & 0 \\
0 & 1
\end{array}\right)=\left(\begin{array}{cc}
D & S V \\
V^{*} S^{*} & u_{n \bar{n}}
\end{array}\right) .
$$

By $\left|u_{j \bar{n}}\right|,\left|u_{n \bar{j}}\right|<C$ for $j<n$ and $\chi_{i \bar{j}}>0$, we may assume that $u_{n \bar{n}}$ is so large (otherwise we are done) that $g_{i \bar{j}}=\chi_{i \bar{j}}+u_{i \bar{j}}(0)>0$, i.e., positive definite. So

$$
\lambda_{i}(A)>0
$$

for every $i=1, . ., n$. Hence,

$$
(\operatorname{det} A)^{\frac{1}{n}} \leq C_{m, n}\left[S_{m}(\lambda(A))\right]^{\frac{1}{m}}=C_{m, n} h .
$$

By $\operatorname{det} g_{i \bar{j}}=\operatorname{det} \alpha_{i \bar{j}} \cdot \operatorname{det} A_{j}^{i}$ we get that $\operatorname{det} g_{i \bar{j}} \leq C$. Since

$$
\left[g_{i \bar{j}}\right]_{i, j<n} \geq\left[\chi_{i \bar{j}}\right]_{i, j<n}>0
$$

and

$$
\operatorname{det} g_{i \bar{j}}=g_{n \bar{n}} \operatorname{det}\left(\left[g_{i \bar{j}}\right]_{i, j<n}\right)+O(1),
$$

we have $g_{n \bar{n}} \leq C$. Thus, the normal-normal derivative bound at a boundary point is proven.

Altogether, we have proven the $C^{2}$-estimate (8.1) and completed the proof of Theorem 1.1 .

\section{Appendix}

The results in this section are classical. It is a natural generalisation of properties of subharmonic functions (see e.g [35]). However, we could not find the the precise forms that we need in the literature. Some of them have been pointed out recently by Harvey-Lawson [31. Our setup here is simpler than the one in [31, therefore we have several finer properties. We emphasize here the use of a theorem of Littman 47. For the readers' convenience we give results with proofs here. 
9.1. Littman's theorem. We briefly recall a simpler version of a result of Littman [46, 47]. Roughly speaking it allows to approximate a generalised subharmonic function (with respect to a uniformly elliptic operator $L$ ) in a constructive way.

Let $D$ be a smoothly bounded domain in $\mathbb{R}^{n}, n \geq 3$. Consider the partial differential operator $L$ defined by

$$
L u=\left(b^{i j} u\right)_{x_{i} x_{j}}-\left(b^{i}(x) u\right)_{x_{i}}
$$

and assumed to be uniformly elliptic there. Its formal adjoint $L^{*}$ is given by

$$
L^{*} v=b^{i j}(x) v_{x_{i} x_{j}}+b^{i}(x) v_{x_{i}},
$$

where coefficients $b^{i j}(x), b^{i}(x)$ are smooth function on $D$.

We say that $u \in L_{l o c}^{1}(D)$ satisfies $L u \geq 0$ weakly if

$$
\int u(x) L^{*} v(x) \geq 0
$$

for any non-negative function $v$ in $C^{2}(D)$ with compact support in $D$. The natural question is to find a sequence of smooth functions $u_{j}$ such that $L u_{j} \geq 0$ and $u_{j}$ decreases to $u$. The usual convolution with a smooth kernel will not give us the desired sequence.

Before stating Littman's theorem let us introduce some notations. We denote by $g(x, y)$ the Green function of the operator $L_{x}$ with respect to domain $D$ and with singularity at $y \in D$; as constructed for example in [53]. The subindex $x$ means that $L$ acts on functions of $x$. The basic properties of $g$ are:

$$
L_{x}^{*} g(x, y)=0 \quad \text { on } D \backslash\{y\}
$$

and

$$
g(x, y)=O\left(|x-y|^{2-n}\right) \text { as } x \rightarrow y .
$$

In particular, $g(x, y) \rightarrow \infty$ as $x \rightarrow y$. Furthermore, let us denote $\Delta=\{(x, x): x \in$ $\bar{D}\}$, then

$$
g \in C^{0}(\bar{D} \times \bar{D} \backslash \Delta) \cap C^{2}(D \times D \backslash \Delta) ;
$$

also $g(x, y)=0$ for $x \in \partial D$ and a fixed $y \in D$. If we denote $r=|x-y|$. Then

$$
g(x, y)=O\left(r^{2-n}\right), \quad g_{x_{i}}=O\left(r^{1-n}\right), \quad g_{x_{i} x_{j}}=O\left(r^{-n}\right) .
$$

Fix a function $p(t)=1-t^{2}$ for $t \in \mathbb{R}$. So $p(0)=1$ and $p(t) \geq \delta_{0}>0$ for $|t|<\delta_{0}$ small enough. It is easy to see that

$$
L_{x}^{*} p(|x-y|)<0 \text { for }|x-y|<2 \delta_{0} \text { and } x, y \in D .
$$

Let $\Phi(t) \geq 0$ be a smooth function supported on $[0,1]$ satisfying

$$
\begin{aligned}
& \Phi(t) \rightarrow 0 \quad \text { exponetially as } t \rightarrow+\infty \\
& \int_{-\infty}^{\infty} \Phi(t) d t=1 .
\end{aligned}
$$

For $\delta>0$ we write $D_{\delta}=\{z \in D: \operatorname{dist}(z, \partial D)>\delta\}$. For $h \geq 0, x \in D, y \in D_{2 \delta}$ we define a function $G_{h}(x, y)$ on $D \times D_{2 \delta}$ by letting

$$
G_{h}(x, y):=0 \text { for }|x-y| \geq 2 \delta,
$$

and for $|x-y|<2 \delta$,

$$
G_{h}(x, y):=\int_{-\infty}^{\infty} \Phi(s-h) \max \{g(x, y)-s p(x, y), 0\} d s .
$$


Notice that $G_{h}(x, y)=0$ for $|x-y| \geq \delta$ and $h \geq h_{\delta}$, where

$$
h_{\delta}:=\frac{1}{\delta_{0}} \max \left\{g(x, y): \delta \leq|x-y| \leq 2 \delta,(x, y) \in D \times D_{2 \delta}\right\} .
$$

Another remark is that

$$
G_{h}(x, y)-g(x, y)=g(x, y) \int_{g / p}^{\infty} \Phi(s-h) d s-p \int_{-\infty}^{g / p} \Phi(s-h) d s
$$

is continuous for $x \in \bar{D}$ and $y \in D_{2 \delta}$ and it belongs to $C^{2}\left(D \times D_{2 \delta}\right)$ as the rate of $g(x, y)$ growing to $\infty$ is polynomially while $\Phi(t) \rightarrow 0$ exponentially. In particular, $G_{h}(x, y) \rightarrow+\infty$ as $x \rightarrow y$ with the same order of growth as $g(x, y)$.

By a direct computation we get that

$$
\frac{\partial G_{h}}{\partial h}=-p \int_{-\infty}^{g / p} \Phi(s-h) d s \leq 0 .
$$

The formula also shows that $\frac{\partial G_{h}}{\partial h} \in C^{2}(D)$ and compactly supported as a function of $x$. Hence,

$$
J_{h}:=\int L_{x}^{*} G_{h} d x=1
$$

for every $h \geq h_{\delta}$. Indeed, by the property [47, 4.f] we have $\lim _{h \rightarrow \infty} J_{h}=1$, and for any constant $c$ we have $L c=0$. Therefore,

$$
\partial J_{h} / \partial h=\int L_{x}^{*}\left(\partial G_{h} / \partial h\right) d x=\int \partial G_{h} / \partial h L_{x} 1=0 .
$$

Since coefficients $b^{i j}(x), b^{i}(x)$ are smooth, we have

$$
G_{h}(x, y)-g(x, y) \in C^{2}\left(D_{2 \delta}\right)
$$

as a function of $y$ uniformly with respect to $x$ (c.f [47, 4.e]). Hence, $G_{h}$ is a Levi function satisfying

$$
L_{x}^{*} G_{h}(x, y)=O\left(|x-y|^{\lambda-n}\right)
$$

for any $0<\lambda \leq 1$ (c.f [53, (8.5) p. 18]). Therefore, for $u \in L_{l o c}^{1}(D)$ and $h \geq h_{\delta}$,

$$
u_{h}(y)=\int u(x) L_{x}^{*} G_{h}(x, y) d x=\int_{|x-y| \leq \delta} u(x) L_{x}^{*} G_{h}(x, y) d x
$$

is well defined. Notice that the support of $G_{h}(x, y)$, as a function in $x$, shrinks to $y$ as $h \rightarrow+\infty$.

We are ready to state a theorem of Littman 47.

Theorem 9.1 (Littman). Let $u \in L_{l o c}^{1}(D)$ be such that $L u \geq 0$ weakly in $D$ in the sense of (9.1). Then, $\left\{u_{h}(x)\right\}_{h \geq h_{\delta}}$, defined by (9.5), are smooth functions satisfying:

- $L u_{h} \geq 0$

- $u_{h}$ is a nonincreasing sequence as $h \rightarrow+\infty, u_{h}$ converges to $u$ in $L^{1}\left(D_{2 \delta}\right)$;

- $U(x):=\lim _{h \rightarrow \infty} u_{h}(x)$ is upper semicontinuous, and $U(x)=u(x)$ almost everywhere. 
9.2. Properties of $\omega$-subharmonic functions. Let $\omega$ be a Hermitian metric on a bounded open set $\Omega \subset \mathbb{C}^{n}$. Let us denote

$$
\Delta_{\omega}:=\omega^{\bar{j} i}(z) \frac{\partial^{2}}{\partial z^{i} \partial \bar{z}^{j}} .
$$

We first recall

Definition 9.2. A function $u: \Omega \rightarrow[-\infty,+\infty[$ is called $\omega$-subharmonic if

(a) $u$ is upper semicontinuous and $u \in L_{\text {loc }}^{1}(\Omega)$.

(b) for every relatively compact open set $D \subset \subset \Omega$ and every $h \in C^{0}(\bar{D})$ and $\Delta_{\omega} h=0$ in $D$, if $h \geq u$ on $\partial D$, then $h \geq u$ on $\bar{D}$.

As in the case of subharmonic functions we have the following properties. These properties are proved by using the above definition (see also [35, Theorem 3.2.2]).

Proposition 9.3. Let $\Omega$ be a bounded open set in $\mathbb{C}^{n}$.

(a) If $u_{1} \geq u_{2} \geq \cdots$ is a decreasing sequence of $\omega$-subharmonic functions, then $u:=\lim _{j \rightarrow+\infty} u_{j}$ is either $\omega-$ subharmonic or $\equiv-\infty$.

(b) If $u, v$ belong to $S H(\omega)$, then so does $\max \{u, v\}$.

Proof. (a) is obvious. We shall prove (b). It is rather standard (see [30]), but probably it is not so well known. We include the proof for the sake of completeness. Observe that

$$
\max \{u, v\}=\lim _{j \rightarrow+\infty} \frac{\log \left(e^{j u}+e^{j v}\right)}{j}
$$

By a simple computation we get that

$$
d d^{c} \log \left(e^{u}+e^{v}\right)=\frac{e^{u} d d^{c} u+e^{v} d d^{c} v}{\left(e^{u}+e^{v}\right)}+\frac{e^{u+v} d(u-v) \wedge d^{c}(u-v)}{\left(e^{u}+e^{v}\right)^{2}} .
$$

It follows that $\frac{1}{j} \log \left(e^{j u}+e^{j v}\right)$ is $\omega$-subharmonic. So is $\max \{u, v\}$.

The subharmonicity is a local notion meaning that a function is subharmonic in a open set if and only if at every point there exists a neighbourhood such that the function is subharmonic in that neighbourhood. The precise statement is

Proposition 9.4. The following statements are equivalent for an upper semicontinuous and locally integrable function $u$ in $\Omega$.

(1) $u$ is an $\omega$-subharmonic function in $\Omega$.

(2) In a neighbourhood $U$ of a given point $a$, if $q \in C^{2}(U)$ such that $q-u \geq 0$ and $q(a)=u(a)$, then

$$
\Delta_{\omega} q(a) \geq 0 \text {. }
$$

Proof. (1) $\Rightarrow(2)$. We argue by contradiction. Suppose that there exist a neighbourhood $U$ of a point $a$ and $q \in C^{2}(U)$ satisfying $q \geq u$ and $q(a)=u(a)$, but

$$
\Delta_{\omega} q(a)<0 .
$$

By Taylor's formula we may assume that $q$ is quadratic and there exists $\varepsilon>0$ such that $\Delta_{\omega} q<-\varepsilon$ on a small ball $B(a, r)$. Solve

$$
\Delta_{\omega} v(z)=-\Delta_{\omega} q, \quad v=0 \quad \text { on } \partial B(a, r) .
$$

Notice that by maximum principle we get that $v(a)<0$. Let $h=v+q$. Then, $\Delta_{\omega} h=0$, and $h \geq u$ on $\bar{B}(a, r)$. However, $h(a)=u(a)+v(a)<u(a)$, which is impossible. The first direction follows. 
$(2) \Rightarrow(1)$. We also argue by contradiction. Suppose that there exist an open set $D \subset \subset \Omega$ and a function $h \in C^{0}(\bar{D})$ and $\Delta_{\omega} h=0$ in $D$, which satisfies $u \leq h$ on $\partial D$, such that $\{u>h\}$ is non-empty. Without loss of generality we may assume that $D$ is a small ball $B$ and $h$ is continuous on $\bar{B}$. Set for $\varepsilon>0$

$$
v_{\varepsilon}(z)=h(z)-\varepsilon|z|^{2} .
$$

Then, the upper semicontinuous function $\left(u-v_{\varepsilon}\right)$ takes its maximum at a point $a \in B$, so

$$
u(z) \leq v_{\varepsilon}(z)+u(a)-v_{\varepsilon}(a) \text { for } z \in B .
$$

By Taylor's formula

$$
\begin{aligned}
h(z) & =h(a)+\Re(P(z))+\frac{1}{2} \frac{\partial^{2} h}{\partial z_{i} \partial \bar{z}_{j}}(a)\left(z_{i}-a_{i}\right) \overline{\left(z_{j}-a_{j}\right)}+O\left(|z-a|^{3}\right) \\
& =: H(z)+O\left(|z-a|^{3}\right),
\end{aligned}
$$

where $P(z)$ is a holomorphic polynomial. Therefore, $\Delta_{\omega} H(a)=0$. Consider the function

$$
q(z)=u(a)-v_{\varepsilon}(a)+H(z)-\varepsilon|z|^{2}+\frac{\varepsilon}{2}|z-a|^{2} .
$$

Then, it is easy to check that $\Delta_{\omega} q(a)<0, q(a)=u(a)$ and $q(z) \geq u(z)$ in a neighbourhood of $a$. This is impossible and the proof is completed.

Since $\omega$-subharmonicity is a local property we easily get the gluing lemma.

Lemma 9.5. Let $U \subset V$ be two open sets. Let $u \in S H(\omega, U)$ and $v \in S H(\omega, V)$. Assume that

$$
\limsup _{z \rightarrow \zeta} u(z) \leq v(\zeta) \quad \forall \zeta \in \partial U \cap V .
$$

Then, $\tilde{u} \in S H(\omega, V)$, where

$$
\tilde{u}= \begin{cases}\max \{u, v\} & \text { on } U, \\ v & \text { on } V \backslash U .\end{cases}
$$

Proof. Consider

$$
u_{\varepsilon}=\left\{\begin{array}{l}
\max \{u, v+\varepsilon\} \text { on } U, \\
v+\varepsilon \text { on } V \backslash U .
\end{array}\right.
$$

If $x \in U$, then there is a small ball $B(x, r) \subset U$. Hence,

$$
u_{\varepsilon}=\max \{u, v+\varepsilon\}
$$

is $\omega$-subharmonic in $B(x, r)$. Similarly, for $x \in V \backslash U$ by the assumption on $\partial U \cap V$, there is $B(x, r) \subset V$ such that $u_{\varepsilon}=v+\varepsilon$ on $B(x, r)$. Thus, $u_{\varepsilon} \in S H(\omega, V)$. Since $u_{\varepsilon} \searrow u$ we can apply Proposition 9.3 getting the lemma.

The proposition above shows that we only need to check the $\omega$-subharmonicity of a function on a small ball, but it is not clear whether a sum of two subharmonic functions is again subharmonic. We shall need another criterion.

By linear PDEs potential theory, e.g. see [53, for any ball $B(a, r)$, there exists a Poisson kernel $P_{a, r}$ for the operator $\Delta_{\omega}$. Namely, for every continuous function $\varphi$ on $\partial B(a, r)$, the function

$$
h(z)=\int_{\partial B(a, r)} \varphi(w) P_{a, r}(z, w) d \sigma_{r}(w),
$$


is the unique continuous solution to the Dirichlet problem

$$
\Delta_{\omega} h(z)=0 \quad \text { in } B(a, r), \quad h=\varphi \text { on } \partial B(a, r),
$$

where $d \sigma_{r}(z)$ is the standard surface measure on $\partial B(a, r)$.

Lemma 9.6. Let $u: \Omega \rightarrow[-\infty,+\infty[$ be a locally integrable upper semicontinuous function. For $\Omega_{\delta}=\{z \in \Omega: \operatorname{dist}(z, \partial \Omega)>\delta\}, \delta>0$, consider the function

$$
M(u, a, r)=\int_{\partial B(a, r)} u(z) P_{a, r}(a, z) d \sigma_{r}(z), \quad a \in \Omega_{\delta},
$$

where $r \in[0, \delta]$. Then, $u$ is an $\omega$-subharmonic function if and only if

$$
u(a) \leq M(u, a, r)
$$

for $a \in \Omega_{\delta}, r \in[0, \delta]$. Furthermore, $M(u, a, r)$ decreases to $u(a)$ as $r$ goes to 0 .

Proof. We first prove that it is a necessary condition. Take $\phi \geq u$ to be a continuous function on $\partial B(a, r)$. Then,

$$
h(z)=\int_{\partial B(a, r)} \phi(w) P_{a, r}(z, w) d \sigma_{r}(w)
$$

satisfies $\Delta_{\omega} h=0$ and $h=\phi \geq u$ on $\partial B(a, r)$. It follows from definition that $h \geq u$ on $B(a, r)$. In particular,

$$
u(a) \leq \int_{\partial B(a, r)} \phi(w) P_{a, r}(a, w) d \sigma_{r}(w) .
$$

As $u$ is upper semicontinuous, we can let $\phi \searrow u$. By monotone convergence theorem we get the desired inequality.

Now we prove the other direction by contradiction. Assume that there exist a relatively compact open set $D \subset \Omega, h \in C^{0}(\bar{D})$ with $\Delta_{\omega} h=0$ and $h \geq u$ on $\partial D$, but

$$
c:=\sup _{\bar{D}}(u-h)>0 .
$$

As $v=u-h$ is upper semicontinuous, $c$ is finite and $F:=\{v=c\}$ is a compact set in $D$. We choose $a \in F$ such that it is the closest point to the boundary $\partial D$. Assume that $\operatorname{dist}(a, \partial D)=2 \delta>0$. Since there exists $x \in B(a, \delta)$ such that $v(x)<c$, so there is $B\left(x, \epsilon^{\prime}\right) \subset\{v<c-\epsilon\} \cap B(a, \delta)$ for some $\epsilon, \epsilon^{\prime}>0$. It follows from $\Delta_{\omega} h=0$ on $D$ that

$$
v(a) \leq M(v, a, r) \quad \forall z \in B(a, r), \forall r \leq \delta .
$$

Notice that in our case $\Delta_{\omega} 1=0$ and

$$
\int_{\partial B(a, r)} P_{a, r}(z, w) d \sigma_{r}(w)=1 .
$$

Integrating from 0 to $\delta$ we get that

$$
\delta v(a) \leq \int_{[0, \delta]} \int_{\partial B(a, r)} v(z) P_{r}(a, z) d \sigma_{r}(z) d r<\delta c .
$$

This is impossible. Thus, the sufficient condition is proved.

For the last assertion, let $0 \leq r<\delta$. Fix a continuous function $\phi \geq u$ on $\partial B(0, \delta)$. As $\Delta_{\omega} h=0$ in $B(a, \delta)$ for

$$
h(z)=\int_{\partial B(a, \delta)} \phi(w) P_{\delta}(z, w) d \sigma_{\delta}(w),
$$


we get that $u(z) \leq h(z)$ on $B(a, \delta)$. Therefore,

$$
M(u, a, r) \leq \int_{\partial B(a, r)} h(w) P_{r}(a, w) d \sigma_{r}(w)=h(a) .
$$

Moreover,

$$
h(a)=\int_{\partial B(a, \delta)} \phi(w) P_{\delta}(a, w) d \sigma_{\delta}(w) .
$$

Letting $\phi \searrow u$, we get the monotonicity of $M(u, a, r)$ in $r \in[0, \delta]$. Moreover, as $u$ is upper semicontinuous,

$$
\lim _{r \rightarrow 0} M(u, a, r) \leq u^{*}(a)=u(a)
$$

where we used the fact above that $\int_{\partial B(a, r)} P_{r} d \sigma_{r}=1$.

An immediate consequence of the last assertion in the above lemma is

Corollary 9.7. If two $\omega$-subharmonic functions are equal almost everywhere, then they are equal everywhere.

We are ready to state a consequence of Littman's theorem, which says that we can always find a smooth approximation for $\omega$-subharmonic functions.

Corollary 9.8. Let $u \in S H(\omega, \Omega)$ and $\Omega^{\prime} \subset \subset \Omega$. There exists a sequence of smooth $\omega$-subharmonic functions $[u]_{\varepsilon}$ decreasing to $u$ as $\varepsilon \rightarrow 0$ on $\Omega^{\prime}$.

Proof. We simply choose a smooth domain $D \subset \Omega$ and $\delta>0$ small such that $\Omega^{\prime} \subset D_{2 \delta}$ and let

$$
[u]_{\varepsilon}(z):=u_{h}(z)
$$

where $u_{h}(z), h=1 / \varepsilon>h_{\delta}$, is defined in Theorem 9.1. As $U(z):=\lim _{\varepsilon}[u]_{\varepsilon}$ is equal to $u(z)$ almost everywhere and $u$ is also $\omega$-subharmonic, it follows from Corollary 9.7 that $U=u$ everywhere.

Corollary 9.9. Let $\left\{u_{\alpha}\right\}_{\alpha \in I} \subset S H(\omega)$ be a family that is locally bounded from above. Let $u(z):=\sup _{\alpha} u_{\alpha}(z)$. Then, the upper semicontinuous regularisation $u^{*}$ is $\omega$-subharmonic.

Proof. By Choquet's lemma one can choose an increasing sequence $u_{j} \in S H(\omega)$ such that $u=\lim _{j} u_{j}$. Then, by Littman's theorem and the notation in Corollary $9.8, \lim _{\varepsilon}[u]_{\varepsilon}=U \in S H(\omega)$ and $u=U$ almost everywhere. As $u_{j} \in S H(\omega)$ we have

$$
u_{j} \leq\left[u_{j}\right]_{\varepsilon} \rightarrow[u]_{\varepsilon} \quad \text { as } j \rightarrow+\infty
$$

uniformly on compact subsets of $\Omega$. It follows that $u \leq U$. By upper semicontinuous of $U$ we have $u^{*} \leq U$. By the formula (9.5) and $J_{h}=1, \lim _{\varepsilon}[u]_{\varepsilon} \leq u^{*}$. Thus, $u^{*}=U$.

Lemma 9.10. Let $u$ be an $\omega$-subharmonic function in $\Omega$. Then,

$$
\Delta_{\omega} u \geq 0
$$

in the distributional sense. Conversely, if $v \in L_{l o c}^{1}(\Omega)$ and $\Delta_{\omega} v \geq 0$ (as a distribution), then there exists a unique function $V \in S H(\omega)$ such that $V=v$ in $L_{l o c}^{1}(\Omega)$. 
Proof. Let $[u]_{\varepsilon}, \varepsilon>0$, be the smooth decreasing approximation of $u$. As $\Delta_{\omega}[u]_{\varepsilon} \geq 0$ and the family weakly converges to $\Delta_{\omega} u$, we get the first statement. Conversely, by Littman's theorem we know that $V(z)=\lim _{\varepsilon \rightarrow 0}[v]_{\varepsilon}(z) \in S H(\omega)$ and $V(z)=v(z)$ almost everywhere. Therefore, we get the existence. The uniqueness follows from the fact that two $\omega$-subharmonic functions are equal almost everywhere.

The following result is rather simple but it is important.

Lemma 9.11. Let $u \in S H(\omega)$. Let $K \subset \subset D \subset \subset \Omega$ be a compact set and an open set. Then,

$$
\int_{K} d d^{c} u \wedge \omega^{n-1} \leq C(D, \Omega)\|u\|_{L^{1}(D)} .
$$

Proof. Let $\phi$ be a cut-off function of $K$ and $\operatorname{supp} \phi \subset D$. Then,

$$
\begin{aligned}
\int_{K} d d^{c} u \wedge \omega^{n-1} & \leq \int \phi d d^{c} u \wedge \omega^{n-1} \\
& =\int u d d^{c}\left(\phi \omega^{n-1}\right) \\
& \leq C(D, \Omega)\|u\|_{L^{1}(D)},
\end{aligned}
$$

where we used that $\phi$ is smooth and has compact support in $\Omega$.

Lemma 9.12. The convex cone $S H(\omega)$ is closed in $L_{l o c}^{1}(\Omega)$, and it has a property that every bounded subset is relatively compact.

Proof. Let $u_{j}$ be a sequence in $S H(\omega)$. If $u_{j} \rightarrow u$ in $L_{l o c}^{1}(\Omega)$, then $\Delta_{\omega} u_{j} \rightarrow \Delta_{\omega} u$ in weak topology of distributions, hence $\Delta_{\omega} u \geq 0$, and $u$ can be represented by an $\omega$-subharmonic function thanks to Lemma 9.10 .

Now suppose that $\left\|u_{j}\right\|_{L^{1}(K)}$ is uniformly bounded for every compact subset $K$ of $\Omega$. Let $\mu_{j}=\Delta_{\omega} u_{j} \geq 0$. Let $\psi$ be a test function such that $0 \leq \psi \leq 1$ and $\psi=1$ on $K$. Then, by Lemma 9.11

$$
\mu_{j}(K) \leq \int_{\Omega} \psi \Delta_{\omega} u_{j} \leq C\left\|u_{j}\right\|_{L^{1}\left(K^{\prime}\right)}
$$

where $K^{\prime}=\operatorname{Supp} \psi$. By weak compactness $\mu_{j}$ weakly converges to a positive measure $\mu$. Let $G(x, y)$ be the Green kernel for the smooth domain $D$, where $K^{\prime} \subset D \subset \Omega$. Consider

$$
h_{j}:=u_{j}(z)-\int G(z, w) \psi \mu_{j}(w) .
$$

Notice that since $G(x, y) \in L^{1}(d \lambda(z))$ and $\psi$ has compact support in $D$,

$$
\int G(z, w) \psi \mu_{j}(w) \rightarrow \int G(z, w) \psi \mu(w)
$$

in $L^{1}$ as $j$ goes to $+\infty$. Therefore, $\Delta_{\omega} h_{j}=0$ in $K$ and $\left\|h_{j}\right\|_{L^{1}} \leq C$. Since

$$
h_{j}(z)=\int_{\partial D} h_{j}(w) P(z, w) d \sigma(w),
$$

it follows that $\left\|h_{j}\right\|_{C^{1}} \leq C$. Then, there exists a subsequence $h_{j}$ converging to $h$ uniformly. Therefore,

$$
h_{j}+\int G(z, w) \psi \mu_{j}(w) \rightarrow u=h+\int G(z, w) \psi \mu(w)
$$

in $L^{1}(K)$ as $j$ goes to $\infty$. 
Lemma 9.13. Let $u_{j}$ be a sequence of $\omega$-subharmonic functions which are uniformly bounded above. If $u$ is an $\omega$-subharmonic function and $u_{j} \rightarrow u$ in $\mathcal{D}^{\prime}(\Omega)$, then $u_{j} \rightarrow u$ in $L_{l o c}^{1}(\Omega)$, and

$$
\varlimsup_{j \rightarrow \infty} u_{j}(z) \leq u(z), \quad z \in \Omega,
$$

(where two sides are equal and finite almost everywhere).

Proof. By Corollary 9.8 for $\varepsilon>0$ small enough,

$$
u_{j} \leq\left[u_{j}\right]_{\varepsilon} \rightarrow[u]_{\varepsilon}
$$

uniformly on compact sets in $\Omega$ as $j \rightarrow \infty$. If $0 \leq \phi \in C_{c}^{\infty}$ then

$$
\int\left([u]_{\varepsilon}+\delta-u_{j}\right) \phi d \lambda(z) \rightarrow \int\left([u]_{\varepsilon}+\delta-u\right) \phi d \lambda(z)
$$

as $j \rightarrow \infty$ and if $\delta>0$ the integrand is positive for $j$ large. Hence,

$$
\varlimsup_{j \rightarrow \infty} \int\left|u-u_{j}\right| \phi d \lambda(z) \leq 2 \int\left|[u]_{\varepsilon}+\delta-u\right| \phi d \lambda(z) .
$$

Since $\varepsilon, \delta$ are arbitrary it follows that $u_{j} \rightarrow u$ in $L_{l o c}^{1}$.

By (9.11) it is easy to see that $\overline{\lim }_{j \rightarrow \infty} u_{j} \leq u$ in $\Omega$. Furthermore, Fatou's lemma gives

$$
\int \varlimsup u_{j} \phi d \lambda \geq \varlimsup \int u_{j} \phi d \lambda=\int u \phi d \lambda,
$$

so we conclude that $\varlimsup_{j} u_{j}=u$ almost everywhere.

Lemma 9.14 (Hartogs). Let $f$ be a continuous function on $\Omega$ and $K \subset \subset \Omega$ be a compact set. Suppose that $\left\{v_{j}\right\}_{j \geq 1} \subset S H(\omega)$ decrease point-wise to $v \in S H(\omega)$. Then, for any $\delta>0$, there exists $j_{\delta}$ such that

$$
\sup _{K}\left(v_{j}-f\right) \leq \sup _{K}(v-f)+\delta
$$

for $j \geq j_{\delta}$.

Proof. Let $\left[v_{j}\right]_{\varepsilon}$ and $[v]_{\varepsilon}$ be decreasing approximations defined in Corollary 9.8 for $v_{j}$ and $v$, respectively. As $v_{j}$ converges to $v$ in $L_{l o c}^{1}(\Omega)$, for any fixed $\varepsilon>0$,

$$
\left[v_{j}\right]_{\varepsilon} \rightarrow[v]_{\varepsilon}
$$

uniformly on compact sets of $\Omega$ as $j$ goes to $+\infty$. Since $v_{j} \leq\left[v_{j}\right]_{\varepsilon}$, we have

$$
\sup _{K}\left(v_{j}-f\right) \leq \sup _{K}\left(\left[v_{j}\right]_{\varepsilon}-f\right)
$$

Let $M:=\sup _{K}(v-f)$. By Dini's theorem $\max \left\{M,[v]_{\varepsilon}(z)-f(z)\right\}$ decreases uniformly to $M$ on $\Omega$ as $\varepsilon$ goes to 0 . Hence, for $\varepsilon>0$ small enough,

$$
\sup _{K}\left([v]_{\varepsilon}-f\right) \leq M+\delta / 2 .
$$

Let us fix such a small $\varepsilon$. By uniform convergence (9.12), for $j \geq j_{1}$

$$
\sup _{K}\left(\left[v_{j}\right]_{\varepsilon}-f\right) \leq \sup _{K}\left([v]_{\varepsilon}-f\right)+\delta / 2 .
$$

Thus, altogether we get the desired inequality.

A direct consequence of this lemma is 
Corollary 9.15. Let $\gamma$ be a real $(1,1)-$ form in $\Omega$. Let $v \in S H_{\gamma, 1}(\omega) \cap L^{\infty}(\Omega)$. Let $\left\{v_{j}\right\}_{j \geq 1} \subset S H_{\gamma, 1}(\omega) \cap L^{\infty}(\Omega)$ be such that

$$
\lim _{j \rightarrow+\infty} v_{j}(z)=v(z) \quad \forall z \in \Omega .
$$

Let $K \subset \Omega$ be a compact set and $\delta>0$. Then, there exists $j_{\delta}$ such that for $j \geq j_{\delta}$,

$$
v_{j}(z) \leq \sup _{K} v+\delta .
$$

Proof. We can find a smooth function $w$ in $\Omega$ such that

$$
d d^{c} w \wedge \omega^{n-1}=\gamma \wedge \omega^{n-1} .
$$

As $u_{j}=v_{j}+w$ and $u=v+w$ satisfy assumptions of Lemma 9.14, we can apply it for $f=w$ to get the statement of the corollary.

Corollary 9.16. Let $\left\{u_{j}\right\}_{j>1} \subset S H(\omega)$ be a sequence that is locally uniformly bounded above. Define $u(z)=\lim _{\sup _{j \rightarrow+\infty}} u_{j}(z)$. Then, the upper semicontinuous regularisation $u^{*}$ is either $\omega-$ subharmonic or $\equiv-\infty$.

Proof. Let $v_{k}=\sup _{j \geq k} u_{j}$. Thanks to Corollary 9.9, $v_{k}^{*} \in S H(\omega)$ and $v_{k}^{*}$ decreases to $v \in S H(\omega)$ or $\equiv-\infty$. Clearly, $v \geq u$, and thus $v \geq u^{*} \geq u$. Since $v_{k}=v_{k}^{*}$ almost everywhere, so $v=u$ almost everywhere. Furthermore, it is easy to see that $\Delta_{\omega} u \geq 0$. By Lemma 9.13

$$
v=\lim _{\varepsilon}[v]_{\varepsilon} \leq \limsup _{\varepsilon}[u]_{\varepsilon} \leq u^{*} .
$$

Therefore, $v=u^{*}$ everywhere.

We now prove that our definition is indeed equivalent to the definition given by Lu-Nguyen [51, Definition 2.3], (see also Dinew-Lu [20]).

Lemma 9.17. A function $u: \Omega \rightarrow[-\infty,+\infty[$ is $\omega-$ subharmonic if and only if it satisfies the following two conditions:

(i) upper semicontinuous, locally integrable and $\Delta_{\omega} u \geq 0$ in $\Omega$.

(ii) if $v$ satisfies the condition $(i)$ and $v \geq u$ almost everywhere, then $v \geq u$ everywhere.

Proof. We first show that it is a necessary conditions. The only thing that remains to be checked is the condition (ii). Pick $v$ satisfying $(i)$ and $v \geq u$ almost everywehre, we wish to show that $v \geq u$ everywhere. As $J_{h}=1$, it follows from the formulas (9.5), (9.10), and the upper semicontinuity of $v$ that

$$
\lim _{\varepsilon \rightarrow 0}[v]_{\varepsilon}(z) \leq v(z) .
$$

Since $[v]_{\varepsilon} \geq[u]_{\varepsilon}$ for $\varepsilon>0$, letting $\varepsilon \rightarrow 0$, we get that $v \geq u$ everywhere.

Suppose that $u$ satisfies $(i)$ and $(i i)$ above. By Littman's theorem $U(z)=$ $\lim _{\varepsilon}[u]_{\varepsilon}=u(z)$ almost everywhere, where $U(z)$ is an $\omega$-subharmonic function, which also satisfies $(i)$. Hence, $u(z) \leq U(z)$ everywhere in $\Omega$. Moreover, using the upper semicontinuity of $u$ as above, we have $u(z) \geq U(z)$ in $\Omega$.

We define the capacity for Borel sets $E \subset \Omega$,

$$
\mathbf{c}_{1}(E)=\sup \left\{\int_{E} d d^{c} v \wedge \omega^{n-1}: 0 \leq v \leq 1, v \in S H(\omega)\right\} .
$$

According to Lemma $9.11 \mathbf{c}_{1}(E)$ is finite as long as $E$ is relatively compact in $\Omega$. 
The quasi-continuity of $\omega$-subharmonic functions was used in [43. We give here the details of the proof. First, the decreasing convergence implies the convergence in capacity.

Lemma 9.18. Suppose that $u_{j} \in S H(\omega) \cap L^{\infty}(\Omega)$ and $u_{j} \searrow u \in S H(\omega) \cap L^{\infty}(\Omega)$. Then, for any compact $K \subset \Omega$ and $\delta>0$,

$$
\lim _{j \rightarrow+\infty} \mathbf{c}_{1}\left(\left\{u_{j}>u+\delta\right\} \cap K\right)=0 .
$$

Proof. Applying the localisation principle [40, p. 7], we assume that $\Omega$ is a ball and $u_{j}=u=h$ outside a neighbourhood of $K$. Let $0 \leq v \leq 1$ be $\omega$ - subharmonic in $\Omega$. We have

$$
\int_{\left\{u+\delta<u_{j}\right\} \cap K} d d^{c} v \wedge \omega^{n-1} \leq \frac{1}{\delta} \int\left(u_{j}-u\right) d d^{c} v \wedge \omega^{n-1} .
$$

By Stokes's theorem,

$$
\begin{aligned}
\int\left(u_{j}-u\right) d d^{c} v \wedge \omega^{n-1}= & -\int d\left(u_{j}-u\right) \wedge d^{c} v \wedge \omega^{n-1} \\
& +\int\left(u_{j}-u\right) d^{c} v \wedge d \omega^{n-1}
\end{aligned}
$$

We shall show that both integrals on the right hand side tend to 0 as $j$ goes to $+\infty$. Hence, we get the lemma. The second one is easier. Indeed, by Schwarz's inequality [55],

$$
\begin{aligned}
\left|\int\left(u_{j}-u\right) d^{c} v \wedge d \omega^{n-1}\right| \leq & C\left(\int\left(u_{j}-u\right) d v \wedge d^{c} v \wedge \omega^{n-1}\right)^{\frac{1}{2}} \times \\
& \times\left(\int\left(u_{j}-u\right) \omega^{n}\right)^{\frac{1}{2}} .
\end{aligned}
$$

Therefore the second integral of the right hand side in (9.14) goes to 0 as $j \rightarrow+\infty$.

Similarly, we use the Schwarz inequality for the first integral in (9.14). Let $K \subset D \subset \subset \Omega$ such that $u_{j}=u$ on $\Omega \backslash D$.

$$
\begin{aligned}
\left|\int d\left(u_{j}-u\right) \wedge d^{c} v \wedge \omega^{n-1}\right| \leq & C\left(\int d\left(u_{j}-u\right) \wedge d^{c}\left(u_{j}-u\right) \wedge \omega^{n-1}\right)^{\frac{1}{2}} \times \\
& \times\left(\int_{D} d v \wedge d^{c} v \wedge \omega^{n-1}\right)^{\frac{1}{2}} .
\end{aligned}
$$

Again by Stokes's theorem

$$
\begin{aligned}
& \int d\left(u_{j}-u\right) \wedge d^{c}\left(u_{j}-u\right) \wedge \omega^{n-1} \\
& =-\int\left(u_{j}-u\right) d d^{c}\left(u_{j}-u\right) \wedge \omega^{n-1}+\int\left(u_{j}-u\right) d^{c}\left(u_{j}-u\right) \wedge d \omega^{n-1} \\
& =\int\left(u_{j}-u\right) d d^{c} u \wedge \omega^{n-1}-\int\left(u_{j}-u\right) d d^{c} u_{j} \wedge \omega^{n-1} \\
& \quad+\frac{1}{2} \int d^{c}\left(u_{j}-u\right)^{2} \wedge d \omega^{n-1} \\
& \leq \int\left(u_{j}-u\right) d d^{c} u \wedge \omega^{n-1}+\frac{1}{2} \int d^{c}\left(u_{j}-u\right)^{2} \wedge d \omega^{n-1}
\end{aligned}
$$


Thus, the fist integral goes to 0 as $j \rightarrow+\infty$ by the Lebesgue dominated convergence theorem. For the second integral we use Stokes' theorem once more

$$
\begin{aligned}
\int d^{c}\left(u_{j}-u\right)^{2} \wedge d \omega^{n-1} & =-\int\left(u_{j}-u\right)^{2} d d^{c} \omega^{n-1} \\
& \leq C \int\left(u_{j}-u\right)^{2} \omega^{n} .
\end{aligned}
$$

The right hand side also goes to 0 as $j \rightarrow \infty$. Thus, we get the lemma.

Lemma 9.19. Let $u \in S H(\omega) \cap L^{\infty}(\Omega)$. Then for each $\varepsilon>0$, there is an open subset $\mathcal{O}$ of $\Omega$ such that $\mathbf{c}_{1}(\mathcal{O}, \Omega)<\varepsilon$ and $u$ is continuous on $\Omega \backslash \mathcal{O}$.

Proof. We may assume that $\Omega$ is a small ball because of the properties of capacity:

- if $E \subset \Omega_{1} \subset \Omega_{2}$, then $\mathbf{c}_{1}\left(E, \Omega_{2}\right) \leq \mathbf{c}_{1}\left(E, \Omega_{1}\right)$.

- $\mathbf{c}_{1}\left(\bigcup_{j} E_{j}\right) \leq \sum_{j} \mathbf{c}_{1}\left(E_{j}\right)$.

Let $S H(\omega) \cap C^{\infty}(\Omega) \ni u_{j} \searrow u$ and fix a compact set $K \subset \Omega$. By Lemma 9.18 there exists an integer $j_{k}$ and an open set

$$
\mathcal{O}_{l}=\left\{u_{j_{l}}>u+\frac{1}{l}\right\} \subset \Omega,
$$

such that $\mathbf{c}_{1}\left(\mathcal{O}_{k} \cap K, \Omega\right)<2^{-k}$. If $G_{k}=\cup_{l>k} \mathcal{O}_{l}$. Then, $u_{j_{k}}$ decreases uniformly to $u$ on $K \backslash G_{k}$. Hence, $u$ is continuous on $K \backslash G_{k}$.

Applying the argument above for a sequence of compact sets $K_{j}$ increasing to $\Omega$ we get open sets $G_{j}$ that $\mathbf{c}_{1}\left(G_{j}, \Omega\right)<\varepsilon 2^{-j}$. Let $\mathcal{O}=\cup_{j} G_{j}$, the lemma follows.

\section{REFERENCES}

[1] E. Bedford and B. A. Taylor, The Dirichlet problem for a complex Monge-Ampère operator. Invent. math. 37 (1976), 1-44.

[2] E. Bedford and B. A. Taylor, A new capacity for plurisubharmonic functions. Acta Math. 149 (1982), 1-40.

[3] E. Bedford and B. A. Taylor, Fine topology, Šilov boundary, and $\left(d d^{c}\right)^{n}$, J. Funct. Anal. 72 (1987), no. 2, 225-251.

[4] R. Berman, From Monge-Ampère equations to envelopes and geodesic rays in the zero temperature limit. preprint, arXiv:1307.3008

[5] Z. Błocki, Weak solutions to the complex Hessian equation, Ann. Inst. Fourier (Grenoble) 55 (2005), no. 5, 1735-1756.

[6] Z. Błocki, A gradient estimate in the Calabi-Yau theorem, Math. Ann. 344 (2009), no. 2, $317-327$

[7] Z. Błocki, On geodesics in the space of Kähler metrics, in Advances in geometric analysis, 3-19, Adv. Lect. Math. (ALM), 21, Int. Press, Somerville, MA.

[8] S. Boucksom, Monge-Ampère equations on complex manifolds with boundary. 257-282, Lecture Notes in Math., 2038, Springer, Heidelberg, 2012.

[9] M. Brunella, Locally conformally Kähler metrics on certain non-Kählerian surfaces, Math. Ann. 346 (2010), no. 3, 629-639.

[10] X. Chen, The space of Kähler metrics, J. Differential Geom. 56 (2000), no. 2, 189-234.

[11] U. Cegrell and S. Kołodziej, The Dirichlet problem for the complex Monge-Ampère operator: Perron classes and rotation-invariant measures, Michigan Math. J. 41 (1994), no. 3, 563569.

[12] L. Caffarelli, J. Kohn, L. Nirenberg and J. Spruck, The Dirichlet problem for nonlinear second-order elliptic equations. II. Complex Monge-Ampère, and uniformly elliptic, equations, Comm. Pure Appl. Math. 38 (1985), no. 2, 209-252

[13] L. Caffarelli, L. Nirenberg and J. Spruck, The Dirichlet problem for nonlinear second-order elliptic equations. III. Functions of the eigenvalues of the Hessian, Acta Math. 155 (1985), no. 3-4, 261-301. 
[14] M. Charabati, Modulus of continuity of solutions to complex Hessian equations, Internat. J. Math. 27 (2016), no. 1, 1650003, 24 pp.

[15] P. Cherrier, A. Hanani, Le problème de Dirichlet pour des équations de Monge-Ampère complexes modifiées. J. Funct. Anal. 156 (1998), 208-251.

[16] P. Cherrier, A. Hanani, Le problème de Dirichlet pour des équations de Monge-Ampère en métrique hermitienne. Bull. Sci. Math. 123 (1999), 577-597.

[17] S. Dinew and S. Kołodziej, Pluripotential estimates on compact Hermitian manifolds. Adv. Lect. Math. (ALM), 21 (2012), International Press, Boston.

[18] S. Dinew and S. Kołodziej, A priori estimates for complex Hessian equations, Anal. PDE 7 (2014), no. $1,227-244$.

[19] S. Dinew and S. Kołodziej, Liouville and Calabi-Yau type theorems for complex Hessian equations. preprint, arXiv:1203.3995, to appear in Amer. J. Math.

[20] S. Dinew and C. H. Lu, Mixed Hessian inequalities and uniqueness in the class $\mathcal{E}(X, \omega, m)$, Math. Z. 279 (2015), no. 3-4, 753-766.

[21] S. K. Donaldson, Symmetric spaces, Kähler geometry and Hamiltonian dynamics, in Northern California Symplectic Geometry Seminar, 13-33, Amer. Math. Soc. Transl. Ser. 2, 196, Amer. Math. Soc., Providence.

[22] P. Eyssidieux, V. Guedj and A. Zeriahi, Viscosity solutions to degenerate complex MongeAmpère equations. Comm. Pure Appl. Math. 64 (2011), no. 8, 1059-1094.

[23] P. Eyssidieux, V. Guedj and A. Zeriahi, Continuous approximation of quasiplurisubharmonic functions, in Analysis, complex geometry, and mathematical physics: in honor of Duong H. Phong, 67-78, Contemp. Math., 644, Amer. Math. Soc., Providence, RI.

[24] J.-X. Fu and S.-T. Yau, The theory of superstring with flux on non-Kähler manifolds and the complex Monge-Ampère equation. J. Diff. Geom. 98 (2008), 369-428.

[25] L. Gårding, An inequality for hyperbolic polynomials, J. Math. Mech. 8 (1959), 957-965.

[26] B. Guan, The Dirichlet problem for complex Monge-Ampère equations and regularity of the pluri-complex Green function, Comm. Anal. Geom. 6 (1998), no. 4, 687-703.

[27] B. Guan, Second-order estimates and regularity for fully nonlinear elliptic equations on Riemannian manifolds, Duke Math. J. 163 (2014), no. 8, 1491-1524.

[28] B. Guan and Q. Li, Complex Monge-Ampère equations and totally real submanifolds. Adv. Math. 225 (2010), no. 3, 1185-1223.

[29] B. Guan and W. Sun, On a class of fully nonlinear elliptic equations on Hermitian manifolds, Calc. Var. Partial Differential Equations 54 (2015), no. 1, 901-916.

[30] V.Guedj and A.Zeriahi, Intrinsic capacities on compact Kähler manifolds. J. Geom. Anal. 15 (2005), 607-639.

[31] F. R. Harvey and H. B. Lawson, Jr., The equivalence of viscosity and distributional subsolutions for convex subequations - a strong Bellman principle, Bull. Braz. Math. Soc. (N.S.) 44 (2013), no. 4, 621-652.

[32] F. R. Harvey and H. B. Lawson, Jr., Dirichlet duality and the nonlinear Dirichlet problem on Riemannian manifolds, J. Differential Geom. 88 (2011), no. 3, 395-482.

[33] F. R. Harvey and H. B. Lawson, Jr., Existence, uniqueness and removable singularities for nonlinear partial differential equations in geometry, in Surveys in differential geometry. Geometry and topology, 103-156, Surv. Differ. Geom., 18, Int. Press, Somerville, MA.

[34] F. R. Harvey, H. B. Lawson Jr., and S. Pliś, Smooth approximation of plurisubharmonic functions on almost complex manifolds, Math. Ann. 366 (2016), no. 3-4, 929-940.

[35] L. Hörmander, Notions of convexity, Progress in Mathematics, 127, Birkhäuser Boston, 1994

[36] Z. Hou, X.-N. Ma and D. Wu, A second order estimate for complex Hessian equations on a compact Kähler manifold, Math. Res. Lett. 17 (2010), no. 3, 547-561.

[37] S. Kołodziej, Some sufficient conditions for solvability of the Dirichlet problem for the complex Monge-Ampère operator, Ann. Polon. Math. 65 (1996), 11-21.

[38] S. Kołodziej, The complex Monge-Ampère equation. Acta Math. 180 (1998), 69-117.

[39] S. Kołodziej, The Monge-Ampère equation on compact Kähler manifolds. Indiana Univ. Math. J. 52 (2003), no. 3, 667-686.

[40] S. Kołodziej, The complex Monge-Ampère equation and pluripotential theory. Memoirs Amer. Math. Soc. 178 (2005), pp. 64.

[41] S. Kołodziej and N.-C. Nguyen, Weak solutions to the complex Monge-Ampère equation on Hermitian manifolds. Analysis, Complex Geometry, and Mathematical Physics: In Honor 
of Duong H. Phong, May 7-11, 2013 Columbia University, New York. Contemp. Math 644 (2015), 141-158.

[42] S. Kołodziej and N.-C. Nguyen, Stability and regularity of solutions of the Monge-Ampère equation on Hermitian manifold. preprint, arXiv: 1501.05749.

[43] S. Kołodziej and N.-C. Nguyen, Weak solutions of complex Hessian equations on compact Hermitian manifolds, Compos. Math. 152 (2016), no. 11, 2221-2248.

[44] N. V. Krylov, Boundedly inhomogeneous elliptic and parabolic equations in a domain. (Russian) Izv. Akad. Nauk SSSR Ser. Mat. 47 (1983), no. 1, 75-108.

[45] S.-Y. Li, On the Dirichlet problems for symmetric function equations of the eigenvalues of the complex Hessian, Asian J. Math. 8 (2004), no. 1, 87-106.

[46] W. Littman, A strong maximum principle for weakly L-subharmonic functions, J. Math. Mech. 8 (1959), 761-770.

[47] W. Littman, Generalized subharmonic functions: Monotonic approximations and an improved maximum principle, Ann. Scuola Norm. Sup. Pisa (3) 17 (1963), 207-222.

[48] H.-C. Lu, Viscosity solutions to complex Hessian equations, J. Funct. Anal. 264 (2013), no. $6,1355-1379$.

[49] H.-C. Lu, Solutions to degenerate complex Hessian equations, J. Math. Pures Appl. (9) 100 (2013), no. 6, 785-805.

[50] C. H. Lu, A variational approach to complex Hessian equations in $\mathbb{C}^{n}$, J. Math. Anal. Appl. 431 (2015), no. 1, 228-259.

[51] H-C. Lu and V-D. Nguyen, Degenerate complex Hessian equations on compact Kähler manifolds, Indiana Univ. Math. J. 64 (2015), no. 6, 1721-1745.

[52] M. L. Michelsohn, On the existence of special metrics in complex geometry, Acta Math. 149 (1982), no. 3-4, 261-295.

[53] C. Miranda, Partial differential equations of elliptic type, second revised edition. Translated from the Italian by Zane C. Motteler, Ergebnisse der Mathematik und ihrer Grenzgebiete, Band 2, Springer, New York, 1970.

[54] N.-C. Nguyen, Weak solutions to the complex Hessian equation. Doctoral thesis (2014), Jagiellonian University (available on researchgate.net).

[55] N. C. Nguyen, The complex Monge-Ampère type equation on compact Hermitian manifolds and applications, Adv. Math. 286 (2016), 240-285.

[56] D. H. Phong, S. Picard and X. Zhang, On estimates for the Fu-Yau generalization of a Strominger system, preprint. arXiv:1507.08193. To appear in J. Reine Angew. Math.

[57] D. H. Phong, S. Picard and X. Zhang, A second order estimate for general complex Hessian equations, Anal. PDE 9 (2016), no. 7, 1693-1709.

[58] D. H. Phong, S. Picard and X. Zhang, The Fu-Yau equation with negative slope parameter, preprint. arXiv: 1602.08838. To appear in Invent. Math.

[59] S. Pliś, The smoothing of m-subharmonic functions, preprint. arXiv: 1312.1906

[60] S. Semmes, Complex Monge-Ampère and symplectic manifolds, Amer. J. Math. 114 (1992), no. $3,495-550$.

[61] J. Spruck, Geometric aspects of the theory of fully nonlinear elliptic equations, in Global theory of minimal surfaces, 283-309, Clay Math. Proc., 2, Amer. Math. Soc., Providence, RI, 2005.

[62] G. Székelyhidi, Fully non-linear elliptic equations on compact Hermitian manifolds. preprint, arXiv:1501.02762 3 . To appear in J. Differential Geom.

[63] G. Székelyhidi, V. Tosatti and B. Weinkove, Gauduchon metrics with prescribed volume form, preprint, arXiv:1503.04491

[64] V. Tosatti, Y. Wang, B. Weinkove and X. Yang, $C^{2, \alpha}$ estimates for nonlinear elliptic equations in complex and almost complex geometry. Calc. Var. Partial Differential Equations 54 (2015), no. 1, 431-453.

[65] V. Tosatti and B. Weinkove, The Monge-Ampère equation for $(n-1)$-plurisubharmonic functions on a compact Kähler manifold, J. Amer. Math. Soc. 30 (2017), no. 2, 311-346.

[66] V. Tosatti and B. Weinkove, Hermitian metrics, $(n-1, n-1)$ forms and Monge-Ampère equations. preprint, arXiv:1310.6326. To appear in J. Reine Angew. Math. 2017.

[67] X. J. Wang, A class of fully nonlinear elliptic equations and related functionals, Indiana Univ. Math. J. 43 (1994), no. 1, 25-54.

[68] Y. Wang, A viscosity approach to the Dirichlet problem for complex Monge-Ampère equations. Math. Z. 272 (2012), no. 1-2, 497-513. 
[69] D. Zhang, Hessian equations on closed Hermitian manifolds. preprint, arXiv:1501.03553

Faculty of Mathematics and Computer Science, Jagiellonian University 30-348 Kraków, Łojasiewicza 6, Poland

E-mail address: dongwei.gu@im.uj.edu.pl, Nguyen.Ngoc.Cuong@im.uj.edu.pl

Department of Mathematics and Center for Geometry and its Applications, Pohang University of Science and Technology, 37673, The Republic of Korea

E-mail address: cuongnn@postech.ac.kr 